\title{
Herbert Busch
}
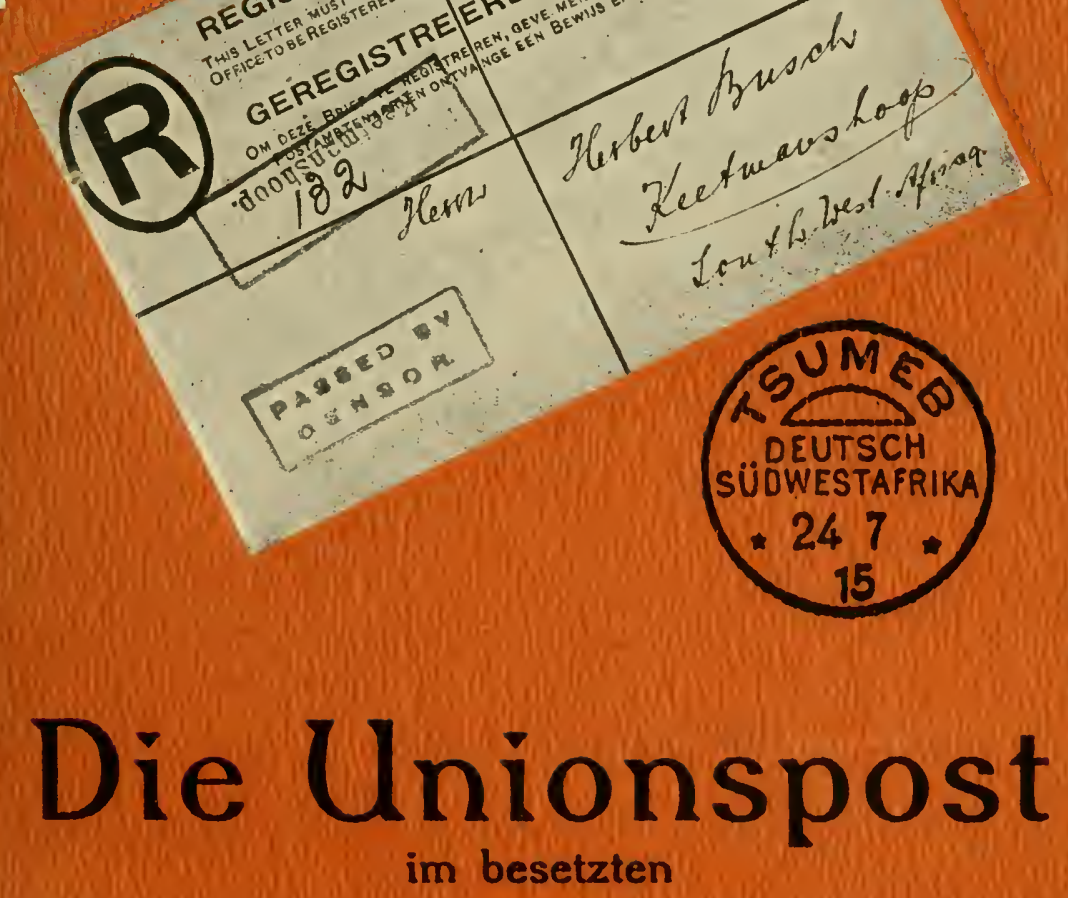

Deutsch $=$ Südwest $=$ Afrika 1914-19

lhre Entwertungen und Stempel deutscher und feindlicher Herkunft.

\section{BAND 1}

Verlag: Briefmarkenhaus Georg Bütow, Berlin C2 Bärohaus Bōrse 



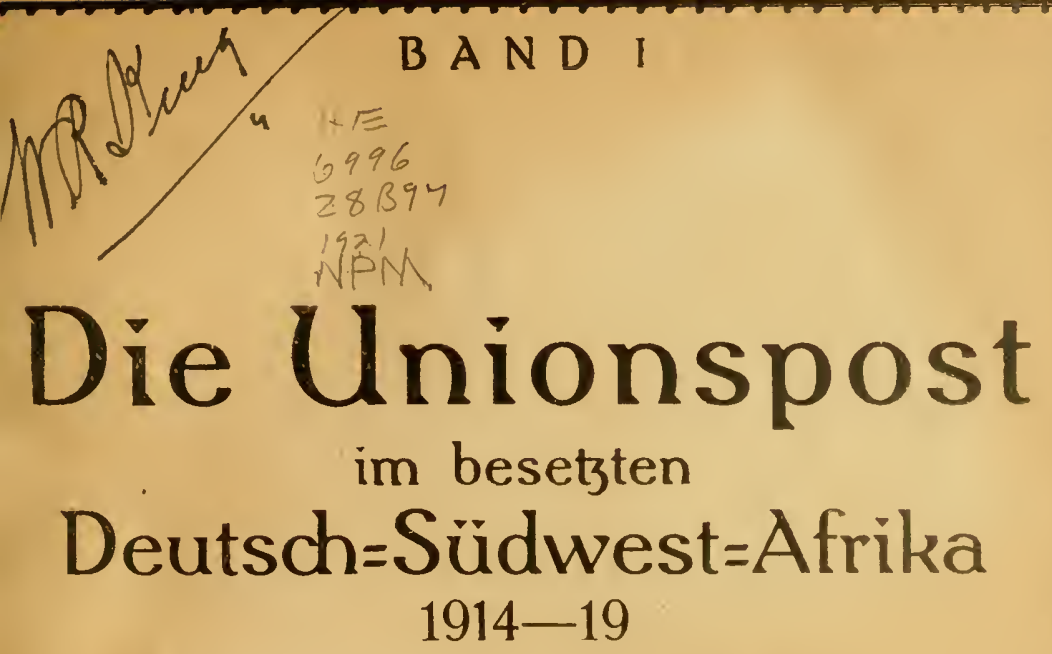

lhre Entwertungen und Stempel deutscher und feindlicher Herkunft

Bearbeitet und zusammengestellt von

Herbert Busch

l. Auflage 1920

Verlag: Briefmarkenhaus Georg Bütow, Berlin C2 Bürohaus Börse 


\begin{abstract}
Alle Rechte,
insbefondere das der Überfetzung vorbehalten.

Copyright 1921. Verlag Georg Bütow, Berlin.
\end{abstract}




\section{Inhaltsverzeichnis.}

Vorwort

politifch=philateliftifche Betrachtung zur Einführung 9-12 Doftanftalten

Albrechts . . . . . . . . . . . . , . . . . 13

Arandis . . . . . . . . . . . . . . . . . 13

Aroab . . . . . . . . . . , . . . . . . 14

Aus

Aus - Rail

Berglands

Bethany

Bradkwaffer

Brackwater

Cauaíokawo

Chamis

Erundu

Feldfchuhhorn

Franzfontein

Garub

Gibeon

Gibeon - Rail . . . . . . . . . . . . 20

Gobabis

Grootfontein

Guchab

Haalenberg.

Ham River - Rail

HatTamas

Kalkfeld - Rail 
Kalkfontein

Kalkrand - Rail

Kanus

Kappsfarm

Karibib

Keetmanshoop

Klein Karas

Klein Windhuk

Kolmannskop

Kub

Kuibis - Rail

Kupferberg

Leutwein - Rail

Lüderitzbucht .

Maltahöhe

Mariental

Mier

32

32

Nakob - Rail

Namutoni

Narubis .

Neuheufis

Okahandja

Okanjande

Okafife - Rail

Okaukueyo

Omaruru

Ondonga

Otavi .

Otavifontein

Otjitambi

Otjiwwarango

Outjo

Rehoboth

Rehoboth - Rail

Rotkuppe

Schweikhardts

Seeheim

Seeis .

Swakopmund 
Tles . . . . . . . . . . . . . . . . 42

Tfumeb . . . . . . . . . 42

Ukamas . . . . . . . . . . . 43

Ulakos . . . . . . . . . . . . 44

Waldau - Rail . . . . . . . . . . . 44

Walvis=Bay . . . . . . . . . . 45

Warmbad . . . . . . . . . 46

Waterberg . . . . . . . . . 46

Wilhelmstal . . . . . . . . . . . . 46

Windhuk . . . . . . . . . . . . . . $47 / 48$

Witvley . . . . . . . . . . . . . . . . 49

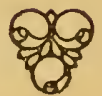





\section{Vorwort.}

Der vorliegende Band zeugt, wie fo manches, von den dauernden Folgen, die den Weltkrieg begleiteten; befonders für Sammler von Abltempelungen und deutโchen Kolonialmarken dürfte diefe Arbeit von lnte= reffe fein. Doch da die Union von Südafrika, deren Verwaltung das Schutzgebiet unterftand, keine Auf= drucksmarken verausgabte, To bleiben die Entwertungen für Deut Ch=Südweftafrika die einzigen philateliftifchen Kriegsurkunden und dürfen aus diefem Grunde einer allgemeineren Beachtung wert fein.

Die Forfchungen waren in der erften Zeit lehr erfchwert durch die unficheren Verhältniffe; teils war die Bahn zerftört, teils wurde noch im nördlichen Ge= biet gekämpfi, daher mögen eine Anzahl erfter und befonders bemerkenswerter Stempel unferer Suche auf immer entgangen fein. Trotzdem ist das gefammelte Material zahlreich und oft felien genug.

Die Vollfändigkeit meiner Arbeit, befonders durch Hinweile auf mir unbekannte Entwertungen, förderte vornehmlich Herr Prof. Adolf Grie $s=W$ indhuk, dem ich hier noch aufrichtig für leine Hinweile danke.

So entftand allmählich das vorliegende Werkchen, dem Inhalt nach ziemlich abgefchloflen, da lärntliche nur erreichbare Quellen herangezogen wurden. Viel= leicht, daß eine fpätere Auflage einige Zuläţe auf: 
weilen könnte. Sollte ein Lefer im Befitze nicht auf= geführter Stempel rein, lo wird freundlichlt gebeten, im Interefle der Philatelie dem Verleger davon Mit= teilung zu machen.

Möge nun diefer Band, ftreng nach Tatfachen bearbeitet, Teine Freude unter den Sammlern finden. Farm Palmental D.S.W.A.

Mủ no dhe $n$, Juni 1920

\section{Herbert Bufch}




\section{Dolitifch-philateliftilche Betrachtung zur Einführung.}

Eine Darftellung der kriegerilchen Vorgānge loll hier weder gegeben werden, noch ift fie auf den fol= genden Seiten verfucht worden. Es handelt fich hier nur darum, einen verftändlichen Uberblick zu gewähren. um die Wege der im Schutzgebiet eindringenden Unions= truppen deutlich zu machen; denn ihren Spuren fols gend, drang die feindliche Poft, mit der wir uns be= falfen, ins Land hinein.

Das Parlament der füdafrikanifchen Union befchloß auf Drängen der britifchen Reichsregierung die Er= oberung Deut [ch=Südweftafrikas. Nur die Stimmen der Nationaliften, der Buren, die die alte Schmach von 1899/02 noch nicht vergeffen hatten, waren gegen diefen plan. lhre Minderheit konnte uns jedoch nicht helfen.

Die erften rafchen Verfuche, Südweft zu nehmen, vermochten deut Cche Truppen am Oranje zu vereiteln. Die lahreszahl 1914 im Buchtitel ift eigentlich nur durch die Befetzung Lüderitzbuchts, der Diamantenftadt, im September 1914 gerechtfertigt. Als Küftenftadt lag fie im Bereich der feindlichen Schiffsgefchütze, diefen ver= danken wir alfo das erfte engliche poltamt. Weil die Zivilbevölkerung der Stadt aber nach Südafrika gefchafft wurde, müßte es richtiger heißen: „Feldpoftamt d. Unions= truppen". Ein Unter[chied kann und darf jedoch Tchwer nützlich fein. Poftttationen wurden faft ausfchließlich an den großen Verbindungsftraßen eröffnet. In den an der Bahn liegenden Orten wurde das Bahnhofs: gebäude meift zum poftamt. Die an diefen Punkten wohnende deutfche Bevölkerung, Coweit fie nicht mit den eigenen Truppen tiefer ins Land flüchtete, durfte fich diefer "Feldpofttation" ebenfalls bedienen. Als 
[päter die Bewohner in ihre Wohnfitze zurückkehrten, außerdem die Referviften fich wieder in friedliche Bürger und Farmer verwandelten, gab es bald mehr Zivil als Militär auf der Doft zu 〔ehen. Die Schalter= beamten, zumeift erft Angehörige der Unionstruppen, wurden fpäter allmählich durch Zivilbeamte erfetzt. Die kleinen Agenturen, meift Dolizeiftationen ange= gliedert, wurden von diefen verwaltet; während die Bahnpoftagenturen den "Stationmafter" der Eifenbahn zum Vorftand hatten. Schließlich überließ die englifche Verwaltung des Schutzgebietes logar einigen Deut tchen die Verwaltung einer Agentur, wie z. B. Neuheufis und Klein=Windhuk. Die Grenzen zwilchen »Feldpofts und Doft, nie lehr ftreng gezogen, vermengten lich lehr bald und vereinigten fchnell zwei fontt unterfchied= liche Begriffe.

Was die Kriegsereignifle betraf, fo ftand der Feind bis Anfang März 1915 meilt nur an den Grenzen des Landes. Der Feind drang im Süden nicht weit über Garub hinaus vor und hatte fich im Norden nur in Ceiner alten Belitzung Walvisbay (Walfilchbucht) feft eingerichtet. Doch (chon Ende März geftalteten lìch die Verhältniffe immer ungünftiger. General Botha übernahm lelbft die Führung, und die in zehn= facher Uberlegenheit an Mannichaften und im Ver= hältnis riefiger und völliger Uberlegenheit an Kriegs= material überall angelammelten Unionstruppen drangen gleichzeitig von faft allen Seiten ins Land hinein. Die Hauptmacht rückte längft der Bahnlinie Swakopmund= Karibib vor, was die Räumung des Südens erforderlich machte. Als Folge flutete der Feind von 3 Seiten her= ein. Vom Weften längs der Bahn über Aus und Kuibis, vom Süden über den Orange nach Warmbad und vom Often über Hafuur. Die beiden letztgenannten Abtei= lungen befetzten den Hauptort des Südens, Keetmans= hoop, am 19. 20. April 1915 und wandten fich fofort nordwärts nach Gibeon. Ein anderer Truppenteil wandte fich von Kuibis nördlich nach Maltahöhe. So war Ende April der gefamte Süden, ein Drittel des Landes, völlig 
befetzt. Ein Teil der deutfchen Bevőlkerung war nach Windhuk und nördlicher geflohen. Aber auch in diefem Ablchnitt drängten die Ereigniffe ralch zur Entlchei= dung. Von Swakopmund, dem Laůfe des Swakop= flußbettes folgend, über Otjimbingue, und von Karibib nach Okahandja entlang der Bahn, drang der Feind, der dann am 12. Mai 1915 die Landeshauptftadt Wind= huk befetzte. Der wichtigfte Teil der Kolonie war verloren, der Rest mußte in kurzer Zeit auch preisge= geben werden. Im Mai und luni zogen fich die deutlchen Truppen unter wachfendem Druck nach Norden zurück, teils längs der Otavibahn, teils am großen Waterberg entlang. In den erften lulitagen ftand das Gros der Verteidiger im Dreieck Otavi $=\mathrm{T}$ sumeb $=$ Grootfontein . Die Südafrikaner hatten dank ihrer zahlenmäßigen Uberlegenheit diefe Aufftellung überflügelt und be= letzten, von Norden umfaflend, die Proviantniederlage Namutoni. Damit war das Schickfal der Schutztruppe beliegelt. Die einfetzenden Verhandlungen führten am 21. Juli 1915 zur Gelamtübergabe. Der Ulbergabe= vertrag wurde in Korab, km 500 der Bahnlinie, unter= zeichnet.

Die aktive Schutztruppe wurde in Aus, in einem befonderen Sammellager, gefangen gehalten; die übrigen Kämpfer durften in ihre Wohnfitze zurück= kehren und ihren Arbeiten im Lande wieder nachgehen. Im Schutzgebiet verblieb eine ftarke, feindliche Polizei= truppe.

Der Poftdienft gewann nun nach und nach wieder feine frühere Ausdehnung, wenn auch die Anzahl der deutfchen Doften noch im Frühjahr 1919 nicht wieder erreicht war.

Da die deut Che Verwaltung ihre Markenbeltände vernichtete oder verfteckte, to fielen dem Feinde falt keine Materialien zu. In Lüderitzbucht hatte ein Ange= höriger der Unionstruppen eine Anzahl Marken mit "Bothaland geftattet. Offiziell wurden niemals deutche Marken in Südweftafrika von der feindlichen Verwaltung mit einem Aufdruck verfehen. 
Die amtliche Bezeichnung, die der Feind unferer Kolonie gab, lautete: "Drotectorate of South Africat. Soweit die Doftftempel einen Landesnamen tragen, lautet er nur: "S. W. Africa".

Zum Schalterverkauf gelangten bei den poftämtern alle gleichzeitigen Marken der Union of South Africa (aus= genommen die $\mathbf{l} \mathscr{E}$ Marke, die jedoch verwendungs= fähig war). Ferner wurden an den Schaltern zuerft die Portomarken von Transvaal verkauft und fpäter nach und nach durch Unionsportomarken erfetzt. Außer= dem gelangten verfchiedentlich ältere Ganzfachen zum Verkauf. Schließlich waren die englifchen "Poftal= orders々 (eine Art Doftcheck) an den größeren Orten zu erhalten.

Neben den Dofttempeln auf Briefen lei noch auf die verChiedenen Stempel für z. B. unabgeholte $p_{o f t=}$ lachen, ungenügend oder nicht frankierte Briefe und Stempel für Einโchreibebriefe hingewiefen. Es genügt, diefe lediglich zu erwähnen, denn belonders auf Ein= Ichreibebriefen herrfchte die buntefte Mannigfaltigkeit. Wo Stempel fehlten, wurde mit verfchiedenen Tinten, mit Blei, oder Tintenftift gewirtfchaftet. An wenigen Orten freilich wurden vorgefundene deut The Einfchreibe= zettel benutzt, diefe werden an Ort und Stelle, wie ähnliche Sonderheiten überhaupt, entfprechend an= geführt.

Damit ift der Stoff des einführenden Teils des Buches erfchöpft. Es lei noch auf die beigegebene Uberfichtskarte aufmerkfam gemacht, die das Verftänd= nis in jeder Weife unterftützt. 


\section{Albrechts. (Johann-Albrechtshöhe.) Poftagentur.}

Bezirk Karibib.

Diefe Poftagentur wurde bereits vor Anfang lu= ni 1915 eröffnet. Der Stempel (Abb. 1) wurde mit dem früheften Datum, 23. September 1916, bekannt. Er liegt ftets in Tehr abgenutzter Form vor. Deutliche, klare Abdrucke waren nicht aufzutreiben und dürften außer= ordentlich Celten fein. Er bekam einen Nachfolger in einem ehemaligen deutfchen Stempel (Abb. 2), dellen Infchriften vom Feinde geändert, bezw. getilgt wurden. Das frühefte Datum feines Vorkommens dürfte der 29. Junì 1917 Cein.

Nb. In Albrechts befand fich 1917 zeitweilig das Lager kriegsgefangener Offiziere der Schutztruppe. Da deren Brieffendungen Portofreiheit genoffen, finden wir den Stempel (Abb. 2) oft auf unfrankierten Briefum= fchlägen.

\section{Arandis. \\ poltagentur.}

Bezirk Swakopmund.

Als Eröffnungs'datum läßt lich nur ein unbeftimmter Tag vor dem 6. Juli 1915 angeben. Vorläufer find un= bekannt. Im Sommer 1916. kam der erfie Stempel (Abb. 3) in Gebrauch. Der Stempel ift mit der erften Albrechtstype verwandt, unterlcheidet fich aber noch durch das hinzugeletzte Wort: "Railk (=Bahn), es handelt fich hier wie in allen fpäteren Fällen um einen

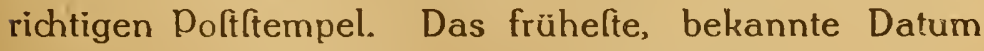
ift der 30. Auguft 1916. Die Stempelfarbe war zuerft Chwarz (Telten) und wurde ab Ende Oktober 1916 blau. Die Poftagentur Arandis=Rail wurde am 1. März 1918 gefchlollen. 


\section{Aroab. \\ poftagentur.}

\section{Diftrikt Aroab (früher Hasuur)}

Leider läßt lich auch hier kein genaues Datum feftftellen, doch fprechen manche Gründe dafür, daß die poft hier Cchon Ende 1915 tätig war. Die Briefe wurden mit dem Doftauto nach Keetmannshoop beför= dert und hier entwertet. Vorläufer dürften daher nicht vorkommen. Mindeftens in der erften Maihälfte 1916 kam der erfte Stempel (Abb, 4) in Gebrauch. Das bekannte frühefte Datum ift der 29. Mai 1976. Die Stempelfarbe war โchwarz. Der Zwi Chenraum im Ortsnamen erklärt fich folgendermaßen: Die Engländer Tandten alle vorgefundenen Stempel nach Windhuk. Es fehlte der Stempel von Aroab. Dagegen fand fich der deutfche Stempel von Arahoab vor. Man entfernte nun die Buchftaben $\mathrm{a} h$ und landte ihn an feinen neuen Beftimmungsort.

Während fonft überall die Kautfchukftempel in fpäterer Zeit durch Metallliempel abgelöft wurden, tauchte in Aroab mit dem zuerft bekannten Datum, 23. September 1916, ein weiterer Stempel auf. (Abb. 5.) Er wurde gleichzeitig mit dem Anfangsftempel gebraucht, nützte fich jedoch [chnell $\mathrm{ab}$, 〔odaß er Ende März 1917 etwa Cchon wieder aus dem Verkehr gezogen wurde. Das Mittelftück diefes Stempels hat ftets fehlerhafte Einftellung. Die Stempelfarbe war immer Ichwarz.

\section{Aus.}

Bezirksamt Lüderitzbucht.

In der Nähe von Aus lag das Lager, in dem die aktive Schutztruppe interniert wurde. Da diefes bereits Ende Juni 1915 gefchah, und die geflüchtete Zivilbe= völkerung zu diefem Zeitpunkt ebenfalls zurückkehrte, fo dürfte der Stempel (Abb. 6) bereits im Auguft benutzt worden lein. Dieler Stempel ift durch ein Merkmal eigenartig, es ift nämlich der einzige Stempel, der zugleich mit dem Landesnamen auch Stundenangabe 
zeigt. Bei allen anderen Stempeln mit Landesnamen fehlen die Stundenangaben, und wo wir lpäter noch Stundenangaben finden, mangelt der Landesname. Die Farbe des Stempels ift ltets Tchwarz.

\section{Aus - Rail. \\ poftagentur. \\ Bezirk Lüderitzbucht.}

Das erfte Entwertungszeichen diefer Station ift als Vorläufer zu betrachten, es handelt fich um einen dreiteiligen Kaftenftempel. (Abb. 7) Die Farbe des Stempels war rötlich-violett. Obgleich eine frühere $\mathrm{B} \dot{\mathrm{e}}=$ nutzung feftteht, war als erftes Datum nur der 25. No= vember $1915 \mathrm{zu}$ ermitteln. Diefer Stempel wurde Ende 1916 aus dem Verkehr gezogen und durch einen Rund= ftempel (Abb. 8) erfetzt. Farbe war ebenfalls rot-violett.

Seit dem 26. Auguft 1917 konnte ein dritter Stempel auf den Briefen feftgeftellt werden. (Abb. 9) Es handelt fich zweifellos um den gleỉchen Stempel wie Abb. 7, doch ift das dritte unnötige Feld weggelaflen worden. Die Farbe war wieder nur rot-violett.

Ein vierter Stempel tauchte am 19. Juli 1918 auf. Er war nur vorübergehend im Gebrauch und dürfte Cehr Celten Cein. (Abb, 10)

Es handelt ficti um einen früheren Dienftftempel des S. A. E. C. (fiehe auch Abb. 58 von Kolmanskop), der nun nach Entfernung der Buchftaben als Doftftempel benutzt wurde. Die Farbe ift bläulich. M it den Buch= ftaben S. A. E. C. kommt der Stempel als Dienftitempel ( $\mathrm{n}$ i ch t Poftftempel) noch am 17. November 1917 auf Briefen vor.

Der endgültige Stempel für Aus - Rail tauchte zuerlt mit dem Datum 25. November 1918 auf. (Abb. 11) Wie erfichtlich, hândelt es fich um den urfprünglichen deutfchen Stempel mit entfprechend veränderten $\ln =$ [chriften. Die Farbe ift Itets Ichwarz. 


\section{Berglands. (Bergland) Doftagentur. Bezirk Rehobot.}

Diele Agentur wurde im Februar 1917 eröffnet und bediente fich logleich eines endgültigen Stempels. (Abb. 12.) Die Stempelfarbe ift immer blaßgrau und macht den Abdruck Tehr unleferlich. Für den 10. und 13. Juni wurde kopfltehendes Tagesdatum feftgeftellt.

Berglands benutzte während einiger Tage aus= hilfsweife, da der obige Stempel zur Reparatur nach Windhuk gefchickt wurde, das poftfiegel als Entwer= tungsitempel. Eine lolche. Entwertung gleicht der von Okafife, (fiehe auch Abb. 90) völlig. Die Farbe ift fchvarz.

\section{Bethany. (Bethanien) poltagentur. Diftrikt Bethanien.}

Die Doft war hier bereits Ende 1915 tätig. Vor= läufer find wahr[cheinlich, jedoch unbekannt. Der Stem= pel (Abb. 13) wurde zuerft mit dem Datum 14. Juni 16 feftgeftellt. Es handelt fich um den urfprünglich deut Chen Stempel, jedoch mit veränderter Endfilbe des Ortsnamens. Die Stempelfarbe ift ltets [chwarz.

Das Datum 25. 10. 1916 hatte kopfftehendes C.

\section{Bradkwaller. poftagentur. Diftrikt Bethanien.}

Der Eröffnungstag der Agentur liegt im Dunkeln. Die Doft ging zumeilf nach Bethanien, wo fie erft entwertet wurde. Es [cheint auch eine proviforifche Entwertung zu geben (Abb. 14), die höchltwahr[chein= lich Bradkwalfer angehört. Sie befindet fich in Tchwarzer Farbe auf einem Einlchreibebrief quer über drei Marken. Diefer Brief ift ordnungsgemäß laut Ankunftftempel in Keetmanshoop am 19. Februar 1916 eingegangen. 
Der endgültige Entwertungsftempel (Abb. 15) wurde mit dem Datum 16. April 1916 zuerft bekannt. Es ift wieder der urfprüngliche deutfche Stempel mit ver= änderter lnfchrift. Die Stempelfarbe ift Chwarz. Es liegt das Fehldatum 9. 4. o h $\mathrm{n}$ e die lahreszahl 18 vor.

Seit dem 8. lanuar 1918 wurde neben dem end= gültigen Stempel noch vorübergehend ein Gummiftempel benutzt (Abb. 16). Wegen völliger Abnutzung wurde diefer bereits Anfang April wieder eingezogen. Die Stempelfarbe ift โchwarz. Diefer Stempel zählt zu den großen Seltenheiten.

\section{Bradkwater.}

Bezirk Windhuk.

Der Uberfchrift wurde ablichtlich weder die Be= zeichnung Doftagentur noch fonft etwas beigefügt. Es kommt eine handfchriftliche Entwertung vom 10. Juni 17 vor, welche, trotz Ankunftitempel auf den betreffenden Doft [achen, zu Unrecht befteht. Brackwater war offi= ziell niemals poftftation. Die Entwertung verdient höchftens als Kuriofum Beachtung. (Abb. 17)

\section{Cauasokawo. \\ poftagentur. \\ Bezirk Outjo.}

Obgleich in einer amtlichen Lifte vom 6. Juli 1916 als Doftation aufgeführt, Tcheint dort nie der Betrieb eröffhet worden zu fein. Eine Entwertung wurde nicht bekannt. In einem fpäteren Verzeichnis fehlt der Name.

\section{Chamis. \\ poftagentur. \\ Diftrikt Bethanien.}

Die Eröffnung der Agentur liegt vor Anfang luli 1916. Vorläufer find unbekannt. Der einzige Stempel (Abb. 18) hatte violette Farbe. Die Abnutzung des Kautfchuk= ftempels erfolgte Cehr [chnell. Im Oktober 1916 erfuhr 
die Stellung des Datums im Stempel eine Veränderung. (Abb. 19) Zugleich wurde die Stempelfarbe von nur ab fchwarz.

Ende Dezember 1915 wurde die Doftagentur Chamis gefchloffen und der Stempel vernichtet. Er ift in beiden Typen Telten.

\section{Erundu. \\ poltagentur. \\ Diftrikt Omaruru.}

Am 30. Juni 1917 wurde diefe Agentur eröffnet und begann ihren Dienft fofort mit einem endgiltigen Stempel (Abb. 20). Der Stempel ifit felten, dá die Agentur bereits 1918 wieder gefchloflen wurde.

\section{Feldschuhhorn \\ Doftagentur. \\ Diftrikt Bethanien.}

Von April und Mai 1916 find amtliche, handfchrift= liche Tintenentwertungen bekannt (Abb. 21). Zur Kon= trolle der Echtheit genügt der Hinweis, daß derartig entwertete Poftfücke immer einen Ankunfttempel tragen müflen, der nur um wenige Tage weiter datiert. Bereits im Sommer 1916 [päteftens wurde die Àgentur Feldfchuhhorn gefchloflen, bevor lie überhaupt einen endgiltigen Stempel erhielt. Diefe Entwertung ift Tehr felten.

\section{Franzfontein Doftagentur. Bezirk Outjo.}

Die Agentur dürfte Teit Frühjahr 1916 beltehen. Vorläû́er find anzunehmen, jedoch nicht bekannt. Der geobäuchliche Stempel (Abb. 22) ift leit dem Datum 28. September 1916 bekannt. Die Stempelfarbe ift fehr abweichend, nämlich immer grün-bläulich. Einfchreibe= 
briefe nahm diele Agentur ausnahmsweile nicht an. Solche Briefe wurden erft nach Outjo gefandt. Es finden fich daher auf Einfchreibebriefen die Stempel von Franzfontein und Outjo nebeneinander vor. - Es liegt nahe zu glauben, daß vor Einführung des Stem= pels alle Briefe aus Franzfontein erft in Outjo entwertet wurden, und eigentliche Vorläufer daher nicht vor= handen find.

\section{Garub.}

poftagentur.

Bezirk Lüderitzbucht.

Obgleich diefer Ort frühzeitig bereits Ende 1914 vom Feinde befetzt wurde, ift nur ein Stempel mit dem Datum 19. Juli 1916 bekannt (Abb. 23). Das Wort "Stations im Stempel zeigt, daß es fich nur um eine Rail Agentur handelt. Farbe war violett=grau. Die Agentur wurde fpäteftens Ende luli 1916 gefchloffen. Der Stempel zählt zu den feltenften des Schutzgebietes.

\section{Gibeon. \\ Doltamit. \\ Bezirk Gibeon.}

Die Poft begann ihre Tätigkeit im April/Mai 1915. Der erfte Stempel ift ein Proviforium (Abb. 24). Er trägt im unteren Feld die Zahl 26, das den urfprüng= lichen Charakter des Stempels als Feldpofttempei kennzeichnet. Als früheftes Datum wurde der 29.6. 15 bekannt. Farbe ausgefprochen blau. Der Stempel war am 26. Juni noch in Gebrauch. Er bekam Ende Juli, fpätenftens Anfang Auguft einen Nachfolger. (Abb. 25) Diefer Stempel ift fehr intereflant. Er war höchftens bis zum Oktober in Gebrauch und wurảe dann endgiltig aus dem Verkehr gezogen. Wir werden ihm in veränderter Form wieder begegnen. Der end= giltige Stempel (Abb. 26) kam im Oktober 1915 in Gebrauch. Die Farbe der beiden Stempel ift tief fchwarz. 


\section{Gibeon=Rail poftagentur. \\ Bezirk Gibeon.}

Diefe Agentur wurde im Bahnhof Gibeon ein= gerichtet, der gegen $10 \mathrm{~km}$ vom Orte entfernt liegt. Die $\mathrm{p}_{\mathrm{o}} \mathrm{ft}$ lachen gingen mit geringen Ausnahmen zum $\mathrm{p}_{\mathrm{oft}}=$ amt Gibeon, wo lie entwertet wurden. Es find jedoch zwei Vorläufer bekannt geworden, Zunächft eine hand= Chriffliche Tintenentwertung (Abb. 27) unter dem 12. Sep= tember 1916. Die andere Abart (Abb. 28) zeigt den Ortsnamen im Typenfatz in violetter Farbe. Ein Datum fehlt. ledoch gibt der nebenftehende Dofttempel von Gibeon darüber Auffchluß, der das Datum 24. Septem= ber 1916 trägt.

Als endgültigen Stempel (Abb. 29) finden wir unferen Bekannten, den Stempel der Abb. No. 25 wieder, jedoch fteht an Stelle des Datums das Wort Rail. Er ift bekannt mit dem erlten Datum 4. April 1917. Die Stempelfarbe ift ftets Ichwarz.

\section{Gobabis. poftagentur. \\ Diftrikt Gobabis.}

Vorläufer lind unbekannt. Die Eröffnung der Agen= tur dürfte auf Ende 1915 fallen. Der endgiltige Stempel (Abb. 30), hat ftets Tchwarze Farbe.

\section{Grootfontein. \\ poftamt.}

Bezirk Grootfontein.

Der Vorläufer (Abb, 31) ift mit dem Datum 30. Juli 1915 bekannt. Es handelt fich wieder um einen urf́prünglichen Militärpoftftempel, der im unteren Feld die Zahl 60 trägt. Die Farbe ift ein blafles Violett. Diefer Vorläufer ift fehr Telten. Der endgiltige Stempel kam gegen Ende 1915 in Gebrauch. (Abb. 32) Die Farbe ift Ichwarz. 


\section{Guchab. \\ poftagentur. \\ Bezirk Grootfontein.}

In Guchab wurde Ende November Anfang Dezem= ber 1916 eine Agentur errichtet. Es gibt keine Vorläufer, denn es konnte einwandfrei feftgeftellt werden, 'daß man die Poftlachen nach $\mathrm{Otavi}$ [chickte, wo fie $a b=$ geftempelt wurden. Seinen Stempel erhielt G u c h a b am 20. lanuar 1917, an welchem Tage er auch zuerft gebraucht wurde. (Abb. 33). Es handelt fich hier um einen zurecht gemachten deutfchen Kreisaus[chnitt= Stempel mit im übrigen geänderter Landesbezeichnung, denn der urfprüngliche Guchabftempel war o h n e Kreisausโchnitt. Farbe ift Ichwarz.

\section{Haalenberg (Tschaukaib) poftagentur. Bezirk Lüderitzbucht.}

Der Eröffnungstag diefer Agentur ließ fich nicht fefttellen. Der einzige bekannte Stempel (Abb. 34) weicht von den übrigen Typen wefentlich ab. Das einzige bekannte Datum ift der 21. Auguft 1916. Farbe des Stempels ift violett. Zu Ende des lahres 1916 wurde die Doftagentur $\mathrm{Ha}$ al e $\mathrm{nberg}$ ge[chloffen. In einer amtlichen lifte vom 9. Dezember 1916 wird fie bereits nicht mehr aufgeführt. Die Station behielt nur den Charakter einer Telegraphen= refpt. einer Tele= fonftation, als welche fie keinen Stempel mehr führte. Der Stempel ift Tehr felten.

\section{Ham River=Rail \\ poftagentur. Diftrikt Warmbad.}

Die Agentur wurde im Herbft 1916 eröffnet. Es gibt keine Vorläufer, Tondern es wurde gleich der neben= 
ftehende Stempel (Abb. 35) in Gebrauch genommen. Die Farbe ift violett. Diefe Agentur wurde Ende 1918 gefchloffen. Der Stempel ift felten.

\section{Hattamas. (Hatzamas) Poftagentur. Bezirk Windhuk.}

Als Vorläufer wäre hier ein Feld= und Telegraphen= poftftempel anzuführen (wie ihn Abb. 65 zeigt). Der Ortsname Hatfamas wurde mit (chwarzer Tinte halb= kreisförmig neben das Datum hineinge Chrieben. Bekannt ift als Datum der 27. Augult 1916. Die Agentur dürfte feit Mitte 1916 beftehen. Die Stempelfarbe ift violett.

Der Stempel (Abb. 36) tritt mit dem Datum 8. Ok= tober 1916 zuerft auf. Die Farbe des Stempels war zuerft violett. Ungefähr feit 5. lanuar 1917 wurde die Stelle des Datums im Stempel geändert. Zugleich wurde die Stempelfarbe feit diefem Zeitpunkt lchwarz. Ab luni 1917 änderte fie fich auffällig blaugrau. Schwarze Stempel diefer Typen find Celien.

Am 11. Auguft 1918 kam als endgiltiger ein früherer deuttcher Stempel in Gebrauch. Es handelt fich nicht um den urfprünglichen deuttchen Stempel, deflen Infchrift anders nämlich Hatzamas lautete. (Abb. 37). Die Stempelfarbe ift blaufchwarz. Die $A b=$ drücke find meíftens fehr undeutlich.

\section{Kabus. \\ poftagentur. \\ Bezirk Keetmanshoop.}

Im Auguft=September 1917 wurde in Kabus ein Genefungsheim für deut Che Militär=Kriegsgefangene eingerichtet. Eine poltagentur wurde eröffnet. Sie benutzte einen ehemaligen deut Then Stempel (Abb. 38). Die Farbe ift Ichwarz. 
In einer amtlichen Lifte vom Frühjahr 1916 wurde

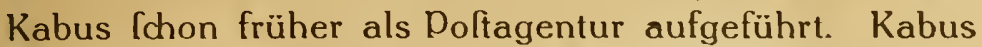
war Teinerzeit Standort englifcher Befałzungstruppen. Von einer damaligen Entwertung ift nichts bekannt geworden. Die Poft dürfte wohl meift nach dem nur 32 Kilometer entfernten Keetmanshoop gebracht worden lein, wo fie abgeftempelt wurde. Die Agentur Kabus wurde endgiltig vor dem 10. Sept. 1918 aufgehoben.

\section{Kalkfeld-Rail. \\ poltagentur. \\ Diltrikt Omaruru.}

Der Poftdienft begann [päteftens im luni 1916. $\mathrm{Ob}$ handfchriftliche oder Ionftige proviforifche Entwer= tungen vorkommen, wird fraglich fein. Der Stempel (Abb. 39) tauchte zuerft mit dem Datum 17. Okt. 1916 auf, die Farbe war zuerft lebhaft violett, die Abdrücke Cauber und deutlich. Später führte die Abnützung des Stempels bis zur Unleferlichkeit, dazu wurde die Stempelfarbe feit 1917 meilt blalggraublau. Stempel= fehler lind häufig. Bekannt ift eine kopfftehende 28 im Datum 28. Februar 1917. Am 29. Oktober und 6. November 1917, Cowie vom 23.-29. Januar 1918 ftanden Datum, Monat und lahreszahl verkehrt. Infolge fehlerhafter Anlage des Stempels ift das Mittelftüdk immer etwas Thief.

Anfang März 1918 kam der endgiltige Stempel in Gebrauch (Abb. 40). Seine Farbe war ein Tehr blaftes Graublau.

\section{Kalkfontein North. \\ poftagentur. \\ Bezirk Gibeon.}

Das frühefte Stempeldatum lautet auf den 4. Sep= tember 1916. Die Farbe des Stempels (Abb. 41) war zuerft violett. Von Mitte September bis Anfang Okto= ber 1916 kommen auch grau Chwarze Entwertungen 
vor, die durchaus feltener find. Der endgiltige Stempel (Abb. 42) wurde unter dem 21. lanuar 1918 zuerft bekannt. Es handelt lich um den urfprünglichen deut Then Stempel vor Kalkfontein=Süd, der entfprechend verändert wurde. Wegen ' Platzmangels wurde aller= dings die Bezeichnung "Northe nur durch ?NTH< an= gedeutet. Die Stempelfarbe war zuerft violettblau, früheltens $a b$ 13. Juli wurden zuerft fchwarze Entwer= tungen bekannt.

\section{Kalkfontein. \\ poftagentur. \\ Bezirk. Warmbad.}

Diefe Agentur bediente fich bereits Ende 1915 des Doftftempels (Abb. 43). Es ift das einzige Entwertungs= zeichen der Station. Die Farbe ift tief fchwarz.

\section{Kalkrand-Rail.}

poftagentur.

Bezirk Gibeon.

Vorläufer Entwertungen find nicht unwahricheinlich. Der vorliegende und einzige Stempel (Abb. 44) kam im Frühjahr oder Sommer 1916 in Gebrauch. Seine Farbe ift blau violett. Im lahre 1916 kommt durch= gehend das Fehldatum $\geqslant 916$ « vor. Es mag zeitweilig mit Tinte in 1916 ergänzt worden fein. Der frühefte Stempelabdruck lautet übrigens auf den 12. Okt. 916. Späteltens etwa Ende April anfangs Mai 1917 wurde die Agentur Kalkrand=Rail endgiltig gefchlolfen.

Die knappe Kursdauer, kaum $3 / 4$ lahr und der unbedeutende Poftverkehr diefer Station haben über= haupt einen der wertvolliten Stempel gefchaffen. 


\section{Kanus. \\ Doftagentur. \\ Bezirk Warmbad.}

Diefe Agentur beftand Teit Ende 1916, führte jedoch keinerleiEntwertungszeichen. Späleftens am 23. Mai 1917 kam der einzige Stempel in Gebrauch (Abb. 45). Die Farbe des Stempels war lchwarz.

N. B. Ende (?) 1917 wurde in Kanus ein Gefangenen= lager für eine Anzahl Deutfcher aus der S. A. Union eingerichtet. Diefe Tatfache erklärt das Vorkommen des Stempels auf unfrankierten Briefen.

\section{Kappsfarm. \\ poltagentur. \\ Bezirk Windhuk.}

Als Eröffnungstag teht ein genaues Datum zur Verfügung. Diefe Agentur begann ihren Dienft am 24. September 1917. Der Stempel (Abb. 46), aus einer unbekannten deut\{chen Type hergeftellt, hat grau= fchwarze Farbe und zeigt ttets fehr undeutliche Abdrücke.

Ein Datumfehler ift lehr merkwürdig. Nicht gerade weil der Punkt zuerft fteht und to die Ziffer "2712" ergibt, Condern weil es fich um den 25. oder 26. Dez. handeln muß. Die betreffenden Doftfücke mit dem Fehldatum zeigen nämlich alle den Durchgangsftempel "Windhoek 26. 12. 17\% und den Ankunftsftempel * Karibib 27. 12. 17. $\quad \mathrm{Da}$ der Poftverkehr von Kappsfarm nicht gerade bedeutend ift, lo trägt der zu allererft in diefer Agentur aufgenommene Einโchreibebrief No. 1 zufällig das Fehldatum.

\section{Karibib. \\ poftamt. \\ Bezirk Karibib.}

Kurze Zeit nach der Befetzung diefer Ortchaft durch die Uniontruppen d. h. am 10. Mai 1915, muß 
der Poftdienft begonnen haben. Der erftbekante Stempel (Abb. 47) führt das Datum 31. Juli 1915. Gerüchtweife foll diefer Stempel anfangs einige Tage mit dem deut Tchen Landesnamen in Gebrauch gewefen fein. Eingezogen wurde der Stempel Ende Auguft 1915 (18. 8. 15. ?) Später kam derfelbe Stempel noch einmal vorübergehend in Gebrauch und zwar von Mitte April 1917 (16.4.) etwa, bis Mitte oder Ende Mai 1917 (18.5.). Diefe Gebraudhszeiten lind Charf getrennt zu halten, weil der Stempel mit der lahreszahl 1917 welentlich Celtener ift. Der Nachfolger war der endgiltige Stempel (Abb.48) Die Farbe diefes und der vorhergehenden Stempel ift Chwarz.

Auf Telegrammen und Telegramm=Briefumfchlägen wurde ein weiterer deut Ther Stempel benutzt (Abb. 49). Diefer tauchte plötzlich vom etwa 10.-19. Auguft 1917 auf Brieffchaften auf, um [päter wieder auf den Tele= grammdienft befchränkt zu bleiben. Er ift daher auf Marken recht Celten.

Nur auf Telegrammen und zwar noch Ende luli 15 führte Karibib einen rotvioletten Stempel wie neben= ftehend (Abb. 50). Der Ortsname Karibib wurde nicht beigedruckt.

\section{Keetmanshoop. poltamt. \\ Bézirk Keetmanshoop.}

Die Stadt wurde am 19./20. April 1915 vom Feinde

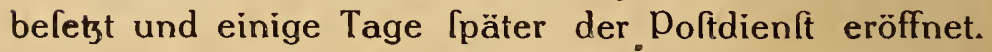
Der Stempel (Abb. 51) hat Thwarze Farbe und trägt im unteren Halbkreis die Nummer 8. Mitte Auguft wurde der Stempel eingezogen. Er ift lehr felten.

Seine Nachfolger waren 2 Stempel, die neben= einander endgiltig in Gebrauch blieben. Zur Ent= wertung der gewöhnlichen Briefe und als Ankunfts= ftempel diente Abb. 52. Für Einfchreibebriefe wurde der Stempel Abb. 53 benutzt. 
Ab 1. Auguft 1917 kommt aus[chließlich auf Tele= grammen noch ein vierter Stempel vor (Abb. 54.) Während die Farbe der obigen Stempel fchwarz ift, ift der Tele= grammftempel ftets violett.

N. B. Das Doftamt Keetmannshoop verwandte im Mai, luni und Oktober 1916 für Einfchreibelendungen den alten deutfchen Einfchreibebriefzettel mit Landes= namen 2Deutfch Südweftafrika". Das Wort "deutfch wurde meift durch!trichen, poftlachen mit diefem Zettel find ziemlich felten.

\section{Klein Karas.}

\section{poftagentur.}

Bezirk Keetmanshoop.

Die Station wurde im Sommer 1916 eröffnet und begann ihren Dienft wahrlcheinlich gleich mit ihrem Kautlchukftempel (Abb. 55.) Die Stempelfarbe war an= fangs lebhaft violett, wurde gegen März 1917 rotviolett.

\section{Klein Windhuk. \\ Doftagentur Windhuk.}

Die Agentur wurde im Herbft 1916 eröffnet und entwertete zuerft handfchriftlich mit blaufchwarzer Tinte. (Abb. 56.) Bekannt ift das Datum 18. September 1916. Der Stempel (Abb. 57.) kommt zuertt im November 1916 vor. Seine Farbe ift Ichwarz.

\section{Kolmannskop. (Kolmannskuppe) poftagentur Bezirk Lüderitzbucht.}

Der längs der Bahnlinie vorrückende Feind befetzte Kolmannskop [chon Ende 1916. Da die Briefe des Fein= des meift nach der Union gingen, dürfte-das 1. Ent= wertungszeichen für unfere Nachforfchung unauffindbar 
fein. Zuerft bekannt wurde ein Kaftenftempel (Abb. 58.) Er enthält den Ortsnamen in deut(cher Schreibweife, nämlich Kolmannskuppe. Links und rechts vom Datum ftehen die Buchftaben "S. A. E. C. (South African En= giner Corps.) Diefer Truppe unterftand der Neubau und Betrieb der Bahn bis Ende 1915. Die Stempelfarbe war violett. Der Stempel war vom 1. Januar 1916 in Gebrauch. Er wurde bald darauf durch den endgiltigen Stempel erfetzt (Abb. 59.) Diefer Stempel foll tatfächlich einige Tage ebenfalls mit der Endfilbe »kuppes in Gebrauch gewefen fein. Die Stempelfarbe war zuerft einige Tage violett und blieb dann endgiltig fchwarz.

Neben dem endgiltigen Stempel wurde fpäter noch einige Zeit der Stempel (Abb. 58) in veränderter Geftalt benutzt. (Siehe Abb. 60) Wie deutlich erkennbar, ift der Stempel durch die Entfernung der Endfilbe "pes und der Buchftaben S. A. E. C. hergeftellt. Die Farbe war blaugrau. Der Stempel ift in der veränderten Geftalt ein Stationsftempel von Kolmannskop Bahn. Seine Ver= wendung auf Marken ift wohl nur als Aushilfe anzufehen

\section{Kub. \\ poltagentur \\ Bezirk Rehoboth.}

Seit Beginn des Frühjahrs 1916 war hier die poft tätig. Der zuerft gebräuchliche Stempel trat 1916 auf. (Abb. 61) Diefer Stempel wurde ohne Datumzufatz be= nutzt. ledoch ift leine Verwendungszeit durch den $A n=$ kunftsftempel zu belegen. Farbe des Stempels ift violett. Er wurde im Herbft 1916 eingezogen. Er ift ziemlich Celten.

Sein Nachfolger (Abb. 62) weift infolge Fehlens der Stempeltype die lahreszahl $\geqslant 916<$ auf. Selten wurde sie durch Hinzufügung einer $\geqslant 7 \%$ mit Tinte ergänzt. Die fehlende Type wurde erft lpäter nachgeliefert, und finden wir dann Anfang 1917 die lahreszahl vollfändig. Die Stempelfarbe ift wieder violett. Klare deutliche Stem= pelabdrucke find ziemlich felten 
Der endgiltige Metallftempel wurde erftmals am 3. Juni 1918 benutzt (Abb. 63.) Zwar handelt es fich um einen deutfchen Stempel, jedoch find die Infchriften der= artig angeordnet und ergänzt, daß eine völlig unge= wohnte Stempeltype entftand. Die Farbe ift Chwarz.

N. B. Die Punkte hinter den Zahlen des Datums kommen auch hochftehend vor.

\section{Kuibis=Rail. \\ poltagentur Keetmanshoop.}

Diefe Agentur muß gegen Mitte 1915 ihre Tätigkeit begonnen haben. Ob der zunächlt folgende Stempel (Abb. 64) das erfte Entwertungszeichen darftellt, ließ fich nicht beftimmt nachweifen. Es handelt fich hier um den ehemaligen deutfchen Kautfchukftempel der RegierungsBohrkolonne-Süd. Von diefer ift Reichsadler und obere Infchrift fortgefchnitten und nur der Ortsname geblieben. Er taucht im luni 1916 zuerft auf in violetter Farbe, und zwar durchwegs ohne Datumzulatz. Mit beigedruck= tem Datum in Typenfatz, dürfte er Tehr felten fein. Ende Auguft wurde auch Cchwarze Stempelfarbe verwandt.

Gegen den 15. Dezember 1916 wurde ein weiterer Stempel in Gebrauch genommen, (Abb. 65) welcher bis Anfang 1917 in Verwendung blieb. Die 3 Buchftaben S. A. R. über dem Datum bedeuten: South African Railways (Süd afrikanilcheEilenbahnen), denn wir haben es da mit einer Rail (Bahn) Doftagentur zu tun. Die Farbe ift violett. Neben diefen ovalen Stempel benutzte man gleichzeitig noch einen kreisrunden Kaut chuk= ftempel (Abb. 66.) Er dürfte ebenfalls 1916 in Gebrauch gekommen Tein. Ende November wurde diefer Stempel auf eigentümliche Weife verändert. Man kam nämlich auf den eigentümlichen Gedanken, die Jahreszahl durd

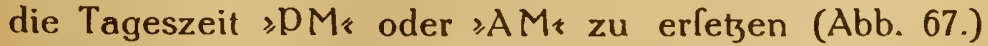
In diefer Anordnung liegen die Daten 24. November 6. Dezember vor. Offenbar Tah man bald-das Sinnlofe diefes Verfahrens ein, denn bereits am 30. Januar 1917 
oder fchon früher kam die lahreszahl wieder zu Ehren. Stempel mit PM oder AM find Telten. Die Farbe war in beiden Typen ftändig Chwarz. Die lełzte Type war noch am 14. Juli 1917 in Verwendung. Sie wurde $[p a ̈=$ teftens im Herbft 7917 durch den endgiltigen Stempel erfetzt. (Abb.68) Die Stempelfarbe ift nunmehr fchwarz. Eine violette Entwertung vom 20. Oktober 1917 ift daher eine Ausnahme und felten.

\section{Kupferberg. \\ Poftagentur. \\ Bezirk Windhuk.}

Man vergleiche was unter Seite 12 über Caualo= Kawo gefagt wurde. Es trifft ebenfalls für Kupferberg zu.

\section{Leutwein - Rail. \\ Poftagentur. \\ Bezirk Windhuk}

Die erfte Entwertung der Poftfachen erfolgte amt= lich mit grüner Tinte. Bekannt if dergeftalt das Datum vom 22. Juni 1916. Vom 22. Juli 1916 liegt die Entwer= tung mit [chwarzer Tinte vor. (Abb. 69) Der Kaut Chuk= ftempel kam im Auguft 1916 in Gebrauch. (Abb. 70). Am 30. Auguft 1916 hat der Stempel Tchwarze Farbe. Diefe ift Tehr felten. Bereits am 10. November 1916, wahrfcheinlich aber auch [chon früher, änderte fich die Stempelfarbe in rotviolett. Die Erhaltung des Stempels blieb durchwegs gut.

Die Agentur Leutwein - Rail wurde gegen Ende 1918 aufgehoben.

\section{Lüderitzbucht \\ poltamt. \\ Bezirk Lüderiţbucht.}

In Lüderitzbucht wurde das erfte feindliche Poftamt im Schutzgebiet überhaupt eröffnet. Die Unionstruppen, 
landeten hier am 19. September 1914. Die Doft begann naturgemäß bald darauf ihren Dienft. Der erfte Siempel (Abb. 71) kam anfcheinend nur auf Einfdhreibebriefen vor. Einen Originalabdruck konnte der Verfaffer nicht erhalten. Der Stempel ift einem Abdruck nachgezeichnet, der von einem Einfchreibezettel ftammt. Da der Zettel die Nr. 296 trug, dürfte der Stempel wohl fchon einige Zeit vor dem 14. Dezember 1914 in Gebrauch gewefen fein.

Schon vorher tcheint ein ovaler Stempel für Ein= fchreibebriefe benutzt worden zu fein. Derfelbe foll im oberen Halbbogen die Inchrift S. Afr. DOST/TEL, in der Mitte ARMY BASE, unten das Datum tragen. Die Farbe war bläulich. Ein Tolcher Stempel befand lich auf einer Einchreibequitturig vom Oktober 1914. Der obenerwähnte Stempel Nr. 71 hat Tchwarze Farbe. Für gewöhnliche Briefe wurde ein Rundftempel benutzt (Abb. 72), der im unteren Halbkreis die Ziffer 4 trägt. Diefer Stempel wurde noch am 9. Juni 1915 benuizî, feine Farbe ift Ichwarz.

Ende Juli 1915 erhielt das Doftamt Ceine beiden endgiltigen Stempel. Abb. 73 wuràe für einfache Briefe, Drucklachen ufw. benutzt, Abb. 74 kommt auf Einfchreibebriefen vor. Farbe ift Ichwarz.

Regelmäßig nur auf Telegrammbriefum[chlägen und Telegrammen, am 23. Dezember 1918 auch als Ankunftsftempel, kommt Abb. 75 vor. Es ift nicht un= möglich, daß mit diefem Stempel auch Poft Tachen ent= wertet wurden. Bekannt ift auch das merkwürdige Fehldatum 11. 2. 5 .

\section{Maltahöhe. poltagentur Diftrikt Maltahöhe.}

Diefe Station wurde gegen Ende 1915 eröffnet. Die erfte Entwertung war ein Typenfatzftempel mit handfchriftlich hinzugefügtem Datum. Belegftücke waren nicht aufzutreiben. Der endgiltige Stempel (Ảbb. 76) 
wurde zuerft am 29. März 1916 beobachtet. Die Stempel= farbe war zuerft violett. Wirklich fchöne, klare Stempel find Tehr felten anzutreffen. Befonders ift das Datum faft ftets unleferlich. Man findet es meift ganz oder teilweise mit Tinte oder Tintenftift ergänzt. Seit etwa Juli 1917 wurde fchwarzblaue Stempelfarbe benützt, wo= durch das Ausfehen des Stempels noch weniger gewann.

\section{Mariental. poftagentur \\ Bezirk Gibeon.}

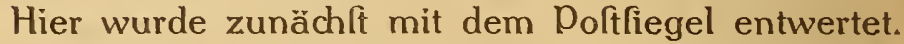
Ein folcher Síempelvorläufer konnte am 21. September 1917 feftgeftellt werden (Abb. 77). Die Farbe war fchwarz. Der endgiltige Stempel kommt bereits am 10. Februar 1916 vor (Abb. 78). Die Farbe ift Tchwarz.

\section{Mier. \\ poltagentur \\ Diftrikt Aroab unterftellt.}

(Mier Territory, Br. Bechuanaland.)

Mier auf britilchem Gebiet gelegen, nahe der deutfchen Grenze, wurde aus praktifchen Gründen der Doftverwaltung des Südweftafrikanifchen Schutzgebietes unterftellt. Eine amtliche Verfügung dieferhalb wurde im März 1917 erlaffen. Die Buchftaben B. B. im Stempel (Abb. 79) bedeuten natürlich British Bechuanaland. Die Stempelfarbe ift Ichwarz.

\section{Nakob=Rail (Nakab) \\ poftagentur Bezirk Warmbad}

Die Agentur wurde gegen Ende 1916 errichtet. Als der Stempel (Abb. 80) am 1. Januar 1917 feftgeftellt wurde, war Teine Farbe blaßblau. Dergeftalt ift er Tehr felten. Am 16. April 1917 war die Farbe bereits violett, und dabei blieb es. Die Abdrücke wurden mit der Zeit [chlechter. Nakob Rail wurde Ende 1918 gefchloffen. 


\section{Namutoni. \\ poftagentur \\ Diftrikt Namutoni.}

Der Doftdienft befteht wahrfcheinlich feit Mitte 1916. Vorläufer find unbekannt. Der Stempel (Abb. 81) taucht zuerft am 30. September 1916 auf. Farbe war violett. Am 1. Juni 1917 zuerft beobachtet, war die Stempel= farbe blaufchwarz, und der Stempel zeigt Spuren von Abnützung.

\section{Narubis. \\ poftagentur \\ Bezirk Keetmanshoop.}

Die Agentur wurde am 9. Juli 1917 eröffnet und zwar gleich mit einem Stempel (Abb. 82). Die Farbe ift violett. Am 17. Oktober 1918 gibt es eine intereffante Fehlerftellung des Datums, nämlich: zuerft der Tag, dann lahr und dann Monat. Alfo 17. 1917 (ftatt 1918) Oct.

\section{Neuheusis. \\ poftagentur \\ Bezirk Windhuk.}

Im Mai 1916 begann diefe Agentur ihren Dienft. Zunächft wurde handlchriftlich entwertet (Abb. 83) Der 19. Mai gilt als erftes Datum. Wenn folche Briefe für Windhuk beftimmt waren, to wurden fie nachträglich auch noch mit dem Dofftempel Windhuk entwertet.

Am 17. Juli, vielleicht aud? Chon früher, tauchte eine neue Entwertung auf (Abb. 84). Poft Neuheusis in kleinem Typenfaty. Es wurde das Datum handfchriftlich mit Tinte beigefügt. Beide Entwertungen find offiziell und recht Telten.

Der erfte Stempel wurde am 9. September 1916 zuerft feftgeftellt (Abb. 85). Seine Farbe ift lchwarz= violett. Im April entftand eine Merkwürdigkeit dadurch, 
daß dem Stempelapparat die Type ADR fehlte. Die fehlende Type wurde handfchriftlich mit Tinte durch die Monatszahl $\geqslant 4$ r angegeben (Abb. 86). Ende des Monats wurde die fehlende Apriltype nachgeliefert und der Stempel mit diefer gebraucht.

Zuerft am 16. Dezember 1918 kommt der endgil= tige Metallftempel von Neuheufis vor (Abb. 87), der aus einem ehemaligen deutfchen Stempel hergeftellt ift. Es handelt fich jedoch nicht um den urfprünglichen Stempel von Neuheufis, was an der veränderten Ein= ftellung der Typen erfichtlich ift. Die Farbe ift grau= fchwarz.

\section{Okahandja. poftamt \\ Diftrikt Okahandja.}

Am 10. Mai 1915 befetzten die feindlichen Truppen diefen Ort, und kurze Zeit darauf muß die Poft ihren Dienft begonnen haben. Zur Verwendung gelangte zunächft ein Stempel, welcher dem Grootfontein Stempel (Abb. 31) gleicht mit dem Unterfchied, daß für Okahandja im unteren Feld die Ziffer 44 gilt. Die Farbe ift blaß= violett und [ehr [chwach, derart, daß die Zahl [elbft felten gut zu erkennen ift. Als erftes Datum konnte der 30. Juli 1915 ausgemacht werden. Am 16. Auguft 1915 war der Stempel noch in Benutzung, fand jedoch fpäteftens im September feinen endgiltigen Nachfolger (Abb. 88). Deffen Farbe ift ftets fchwarz.

\section{Okanjande. poftagentur Diftrikt Omaruru.}

Seit Auguft 1915 befand lich hier eine Agentur, die fich des Stempels (Abb. 89) bediente. Die Farbe war fchwarz. In Okanjande befand fich das Lager der kriegs= gefangenen Offiziere der Schutztruppe. Als diefes Lager im lahre 1917 nach Swakopmund verlegt wurde, ftellte die Poftagentur ihren Dienft ein. Der Stempel ift felten. 


\section{Okafile - Rail \\ poltagentur \\ Diftrikt Okahaindja.}

Die Dolì wurde hier zunächft handfchriftlich entwertet und zwar bis Juni 1916. Derartige Stücke find Tehr felten. Am 21. Juli wurde der erfte Stempel gefehen, deffen Ge= brauch wohl etwas zeitiger fällt. Es handelt fich um den Doltftempel von Okafife (Abb. 90) in Cchwarzer Farbe. Sein Nachfolger wurde zuerft am 5. lanuar 1917 feltge= ftellt. (Abb. 91) Die Farbe ift violett. Die fpäteren Stem= pelabdrüdke waren fehr abgenützt und unleferlich.

\section{Okaukuejo. (Okaukwejo) \\ poftagentur Diftrikt Okaukuejo.}

Die Agentur dürfte im Herbft 1916 eröffnet worden fein. Der einzige bekannte Stempel (Abb. 92) kommt zuerft unter dem 30. Dezember vor. Die Farbe ift grau= blau. Ab Mitte 1917 wurde die Stellung des Datums ver= ändert. Es kamen nur fpärlich Stempel in Umlauf.

\section{Omaruru. poltamt Diltrikt Omaruru.}

Das Poftamt wurde frühzeitig im Mai oder luni 1915 eröffnet. Das erftbekannte Datum ift der 21. Juli 1915. (Abb. 93) Die Doftamtnummer für Omaruru im unteren Feld lautet auf Nr. 50. Die Farbe ift blau. Bekannt ift der 13. Auguft 1915 als letztes Datum. Der endgiltige Nachfolger (Abb. 94) kam Tpäteftens im September 1915 in Gebrauch. Die Farbe ift frhwarz.

Ungefähr leit Mitte Oktober 1918 (28. X. 18) verwen= dete Omaruru den ehemaligen deuttchen Stempel in ver= änderter Geftalt (Abb. 95) befonders auf Telegrammen und Telegrammbriefumfchlägen. Er ift jedoch auch häu= fig auf Marken zu fehen. Auch hier ift die Stempelfarbe ¡chwárz. 


\section{Ondonga. \\ poftagentur \\ Aimboland.}

Wir haben es hier mit der nördlichften pofttation des Schutzgebietes zu tun. Sie begann ihren Dienft im Herbft 1916 mit ihrem endgiltigen Stempel. (Abb. 96) Die Farbe ift violett. Der Stempel ift verhältnismäßig Celten.

\section{Otavi. \\ poltamt \\ Bezirk Grootfontein.}

Das Amt wurde 1975 eröffnet. Anfcheinend war Otavi zuerft Poftagentur und wurde am 11. November 1976 zum Poftamt erhoben. Vorläufer dürften vorhanden (ein, waren aber nicht zu ermitteln. Der Stempel kam ungefähr im September 1915 in Gebrauch. (Abb. 97) Die Farbe ift Tchwarz.

Vorübergehend fcheint in Otavi ein weiterer Stem= pel benutzt worden zu lein (Abb. 98). Es handelt fich um den ehemaligen deut fchen Eîlenbahn-Dienfttempel, der unverändert benutgt wurde. Er befand fich auf ei= nem Briefe mit dem Datum 17. September 1916. Ob es fich hier um den eigentlichen Vorläufer handelt, konnte nicht feftgeftellt werden. Auf Anfrage wurde mitgeteilt, daß diefer Stęmpel in Otavi Mitte lanuar 1917 weder benutzt wurde, noch bekannt war. Die Farbe war fchwarz.

\section{Otavifontein. \\ poltagentur \\ Bezirk Grootfontein}

Der Eröffnungstag dürfte auf Ende 1915 fallen. Der erfte Stempel (Abb. 99) wurde am 9. April 1916 feltge= ftellt. Er trägt im unteren Feld die Ziffer 59. Die Stem= pelfarbe war rotviolett und Ende September fchwarz. Zu diefer Zeit war die Abnützung des Stempels bereits der= 
art yorge[chritten, daß jede Āhnlichkeit mit der früheren Geftalt verloren gegangen war. Die Ziffer 59 war voll= kommen unleferlich. Seit Anfang Oktober 1916 wurde der Name Otavifontein im oberen Feld mit roter Tinte hinzugefchrieben. In diefer Geftalt (Abb. 100) lah man den Stempel zuerft am 18. Oktober 1916.

Diefe unzulängliche Entwertung fand am lahres= ende 1916/1917 ihren endgiltigen Nachfolger, (Abb. 101) deffen Farbe graulchwarz ift. Der 24. Januar 1917 gilt vorläufig als früheftes Datum.

\section{Otjitambi. \\ poftagentur \\ Bezirk Outjo}

Man vergleỉche die Bemerkung zu Cauasokawo.

\section{Otjiwarongo. \\ poftamt \\ Diltrikt Omaruru.}

Kurze Zeit nach der Befełzung dürfte im luni 1915

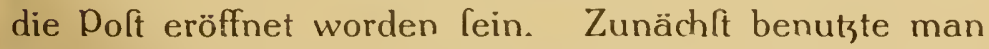
einen Kaftenftempel in bläulicher Farbe (Abb. 102) der im unteren Felde die Nr. 57 trägt. Das frühefte Datum ift der 27. Auguft 1915, am 12. Februar 1916 war der Stempel noch im Gebrauch. Zeitweilig wurde der Orts= name Otjiwarongo in kleinem Typenfats dem Stempel beigedruckt. Dies gefchah am 20.-23. November 1915 und 8. lanuar 1916 (Abb. 103). Der Wert des Stempels wird dadurch beträchtlich erhöht. Neben diesem Stempel war noch ein weiterer im Gebrauch (Abb. 104), deffen Farbe graublau oder grau war. Diefer Stempel mit beigedruckten Ortsnamen Otjiwarongo im Typenfaty, der etwas größer ift als der obenerwähnte Typenlaţ des vorhererwähnten Stempels kommt am 10. und 11. No= vember 1915 vor, ferner am 6. März 1916, und zwar mit Ortsnamenbeidruck bei der Marke, - rückeeitig mit dem gleichen Datum, jedoch o h n e Ortsnamenbeidruck. 
Der Stempel (Abb. 104a) kommt unter dem 3, März 1916 ohne Ortsnamenbeidruck auf Marken vor und ift dann Cehr felten.

Der endgiltige Stempel kam im Frühjahr 1916 in Gebrauch. Der 3. April 1916, wurde als früheftes Datum ermittelt. (Abb. 105) Es ift auffallend, daß diefer Stem= pel keinen Landesnamen aufweift, obwohl im unteren Halbkreis Plaţ genug dazu wäre. Die Farbe diefes Stempels ift ftets Chwarz.

\section{Oüjo. poltamt. \\ Bezirk Outjo.}

Die Eröffnung der Agentur fällt in die Herbftmo= nate 1915. Die erfte Entwertung ift ein Vorläufer (Abb. 106) in blauer Farbe. Die Gebrauchsdaten laufen etwa vom 11. Dezember 1915 bis 13. April 1916. Am 25. April 1916 kommt der Stempel auch in Chwarzer Farbe auf Briefftücken vor.

Eine weitere Entwertung von Outjo kommt am 5. April 1916 vor. Unter dem 6. Mai 1916 wird eine dritte Entwertung bekannt (Abb. 108). Beide proviforifche Ertwertungen haben fchwarze Stempelfarbe und find Cehr Telten.

Nun kam zunächlt ein endgiltiger deutfcher Stem= pel in Gebrauch, der zuerft mit dem 27. Mai 1916 vor= liegt. (Abb. 109) Er wurde aber am 11. lanuar 1917 wieder eingezogen und durch den urfprünglichen deut= Tchen Stempel von Outjo erfetzt (Abb. 110). Diefer trägt den Ortsnamen in etwas kleineren Typen wie fein Vor= gänger. Die Farben diefer beiden Stempel find fchwarz. 


\section{Rehoboth. poltamt. \\ Diftrikt Rehoboth.}

Das Poftamt wurde hier Chon wahrfcheinlich Ende 1915 eröffnet und mag es Vorläufer gegeben haben. Bekannt ift nur der endgiltige Stempel (Abb. 111). Seine Farbe ift Thwarz.

\section{Rehoboth-Rail \\ poftagentur. \\ Diftrikt Rehoboth.}

Die Agentur dürfte im Sommer 1916 eröffnet Cein. Der erfte Stempel (Abb. 112) trägt das Datum 18. Sep= tember 1916. Die Farbe war violett. Am 12. Mai 1917 kommt [chwarzgrüne Stempelfarbe vor und am 6. Juni 1917 war die Stempelfarbe ausgefprochen graufchwarz. Gute klare Stempel kommen wenig vor. Der endgiltige Stempel muß Thon einige Zeit vor dem 20. Februar 1919 benutzt worden fein (Abb. 113). Seine Farbe ift Ichwarz.

\section{Rotkuppe-Rail \\ poftagentur \\ Bezirk Lüderiţbucht.}

Von Rotkuppe ift nur eine Entwertung bekannt (Abb. 114) Der Stempel trägt das Datum 26. April 1916. Es ift aber Teltfam, daß der Durchgangsftempel Lüde= ritzbucht das Datum 30. Juli 1916 trägt und der Ankunfts= ftempel Keetmanshoop auf den 2. Auguft 1916 lautet. Offenbar handelt es fich um ein Fehldatum. Es müßte wohl 26. Juli ftatt April heißen. Die Stempelfarbe ift violett. Die Agentur Rotkuppe wurde im Herbft 1916 gefchloffen. Der Stempel (Abb. 114) zählt zu den. erften Seltenheiten! 


\section{Schweikhardts (Schweikhardtsbrunn) poftagentur. Bezirk Outjo.}

Von Schweikhardtsbrunn liegt eine verbürgt echte handlchriftliche Entwertung vom 28. Juli 1916 vor. (Abb.115) Während der offizielle Namen "Schweikhardts" lautet, hat der dienftuende Beamte einfach die $A b=$ kürzung "S'bronn" angewandt. Da die Agentur im Herbft 1916 gefchloffen wurde, war die Agentur höchftens 3-4 Monate im Betrieb. In den meiften Fällen dürfte die poft in Outjo entwertet worden Cein.

Es befteht alle Urfache, diefe Entwertung für die feltenfte des Schutzgebietes zu halten!

\section{Seeheim. \\ poftagentur.}

Diftr. Bethany (Bethanien)

Voriäufer wahrfcheinlich, doch unbekannt. Der endgiltige Stempel (Abb. 116) kam im Auguft 1915 in Gebrauch. Seine Farbe ift ftets [chwarz.

\section{Seeis. \\ poftagentur. \\ Bezirk Windhuk.}

Der erfte Stempel von Seeis trägt das Datum 3. Oletober 1916 (Abb. 117). Die Farbe ift lchwarz. Ob= gleich die Agentur im Frühjahr 1916 eröffnet worden Cein dürfte, war keine frühere Entwertung feftzuftellen. Diefer Stempel dürfte außerordentlich 〔elten Cein.

Als weitere Entwertung wurde am 2. November 1916 ein neuer Stempel bekannt (Abb. 118). Die Farbe ift fchwarz. Am 10. lanuar 1917 kommt ausnahmsweife violette Farbe vor. Es finden fich auch handlchriftlich verbefferte Daten vor. 
Der dritte endgiltige Stempel tritt am 1. März 1918 zuerft auf. Es handelt fich um einen zurechtgemadhten urfprünglichen deutfchen. Stempel. Die Farbe ift ftets fchwarz. (Abb. 119).

\section{Swakopmund. poftamt. \\ Bezirk Swakopmund.}

Der 1. Stempel kam bei Eröffnung der poft im März 1915 in Gebrauch (Abb. 120). Er trägt im unteren Feld die Feldpoftziffer $\mathrm{Nr}$. 13. Die Farbe ift Cchwarz. Das annähernd letzte Gebrauchsdatum dürfte der 19. Au= guft 1915 Cein. Mit dem 25. Auguft kommt Metallftem= pel in Benutzung. (Abb. 121). Merkwürdigerweile zog man den Stempel anfangs März 1916 wieder aus dem Verkehr, anโcheinend, weil ihın die Stundenangabe fehlte.

Späteftens am 7. März kam der endgiltige $\mathrm{Nach}=$ folger in Gebrauch (Abb. 122). Es handelt fich um einen ehemaligen deutfchen Bahnpofttempel, der fich bedeut= Came Änderungen hat gefallen laflen müifen. Die Farbe des Stempels ift Ichwarz. Während diefer Stempel auf einfachen Briefen und Druckfachen vorkommt, wurde für Einfchreibebriefe ein weiterer urfprünglich deutfcher Stempel in Gebrauch genommen (Abb. 123). Das erft= bekannte Datum ift der 28. März 1916.

Ein Stempel (Abb. 124), der jedoch ausfchließlich auf Paketen vorkommt, wurde ungefähr vom 22. Mai - 15. Juni 1916, Co lauten die feftgeftellten Daten, ge= braucht. Die Farbe ift blutrot. Es handelt fich um das übrig gelaffene Innenoval eines früheren englifchen Dienftftempels. Der Stempel ift außerordentlich felten.

Nur auf Telegrammen und Telegrammbriefum= Tchlägen kommt ein Ovalftempel (Abb. 125) vor.

NB. Der deutfche Einfchreibezettel von Swakop= mund wurde im März, April, Mai, Juni, Juli, Oktober und November 1916 von der englifchen Verwaltung auf= 
gebraucht. Das Wort "deutfch" in der alten Landesbe= zeichnung wurde meift unleferlich gemacht. Ein anderer deutTher Einfchreibezettel ohne Vordruck des Ort und Landesnamens wurde im Auguft 1917 benutzt. Der Orts= name "Swakopmund" wurde in violettem Typenfats hineingedruckt. Auch der deut The Paketzettet wurde Ende 1916 und März 1917 zeitweife teils mit, teils ohne alte durchftrichene Landesbezeichnung gebraucht.

\section{Tses. \\ poltagentur. \\ Bezirk Keetmanshoop.}

Der Eröffnungstag dürfte im Frühjahr 1916 liegen. Es find 2 handCchriftliche Entwertungen bekannt gewor= den. (Abb. 126 und 127.) Der 31. Mai 1916 gilt wohl als erftes, der 30. luni 1916 als letztes gefchriebenes Datum. Beide Handrchriften find Tehr Telten, befonders Handfchrift Nr. 127.

Am 1. Auguft 1916 kommt in Chwarzer Farbe ein Stempel im kleinen Typenfatz vor (Abb.128). Er ift außer= ordentlich felten. Etwa am 5. September 1916, wahr= Tcheinlich früher, bis 28. März 1917 oder Anfang April kam ein weiterer Stempel in Gebrauch (Abb. 129). Die Farbe ift Ichwarz. Guterhaltene Abdrücke lind außer= ordentlich Telten, da die Abnützung des Stempels Tehr rafch erfolgte.

Der endgiltige Stempel von $\mathrm{T}$ s e s tauchte gegen den 25. April 1917 auf. (Abb. 130). Es handelt fich um einen urfprünglich deutfchen Stempel in fchwarzer Farbe.

\section{Tsumeb. \\ poftamt}

Bezirk Grootfontein.

Am 6. Juli 1915 befetzte der Feind T s u m e b, um einige Tage darauf das poftamt wieder zu eröffnen. Der Gegner foll zunächft den deutfchen Stempel kurze 
Zeit unverändert weiter gebraucht haben. Dann machte man zunächlt das Wort »deutfch" im alten Landesna= men dergeftalt ungiltig, indem die Buchftaben in zwei Teile gefchnitten wurden, fodaß nur die obere und untere Hälfte ftehen blieben. (Abb. 131.) In diefer Ausführung ließ man den Stempel vom 24. Juli 1915 bis Mitte Auguft in Verkehr, um ihn dann einer erneuten Änderung zu unterwerfen. Nun entfernte man die Refte des Wortes "deutfch" völlig und in diefer endgiltigen Geftalt ver= wandte man den Stempel bis zum 22. September 1915. Die Farbe des Stempels blieb während der Verwand= lungen ftändig Cchwarz.

Von Anbeginn an war aber noch ein zweiter deut= fcher Stempel in Benutzung. Einmal während der Zeit, da der vorhergehende Stempel zwecks feiner Verände= rung fortgefchickt war, außerdem aber auch neben ihm. doch anfcheinend nur bis Ende Auguft. Es handelt fich in diefem Falle um den deutfchen Feldpoftempel 1 (Abb. 132), der in deutlcher Hand zuletzt in Otavifontein be= nukt wurde, bevor man ihn nach Tsumeb fchickte. Die Landesbezeichnung blieb unverändert. Die Farbe des Stempels war immer fchwarz-violett. Sämtliche bis= her gebrauchte Stempel von Tsume b find fehr felten. befonders Abb. 132.

Der endgiltige Stempel von Tsumeb kam am 23. September 1915 in Gebrauch. (Abb. 133) Seine Farbe iff Cchwarz.

\section{Ukamas. Poltagentur. Bezirk Warmbad.}

Obgleich nach amtl. Ausweis Ukamas am 26. Sept. 1916 eröffnet wurde, tauchte ein Stempel zuerft mit dem Datum 10. Juli 1917. auf. (Abb. 134.) Handfchriftliche Entwertungen follen vorkommen. Wir haben es mit dem alten deutfchen Stempel zu tun, der entfprechende Ver= 
änderungen in der Landesbezeichnung aufweift. Die Farbe des Stempels war bis zum 14. Juli 7917 blau, am 31. Juli fchwarz und am 11. Auguft 1917 wiederum blau. Das Datum 17. Sept. 1917 dagegen weift endgil= tig fchwarze Farbe auf und dabei blieb es.

\section{Uáakos.}

poltamt

Bezirk Karibib.

Wahrrcheinlich führte Urakos einen Vorläufer in Art der Kaftenftempel mit Ziffer. Der endgiltige Stempel (Abb. 135) dürfte noch im Herbft 1915 in Gebrauch ge= kommen Cein. Vom 4. Dezember 1916 bis etwa 7. Mai 1917, dies find die ermittelten Daten, kommt auch der alte deutCche Stempel, (Abb. 136) jedoch ohne Landes= namen auf einfachen Briefen vor, zum Teil auch mit feh= lender lahreszahl. Nach diefer Zeit kommt der Stem= pel auslchließlich auf Telegrammbriefen vor. Die Farbe beider Stempel ift immer [chwarz.

NB. Im März und Mai 1916 (wahr[cheinlich auch [chon lanuar, Februar und April) kommt der deut Tche Einfchreibezettel mit den Ortsnamen U Cakos auf= brauchsweile vor. Derartige Briefe lind nicht häufig.

\section{Waldau-Rail \\ poftagentur Diftrikt Okahandja.}

Diefe Agentur wurde vor Anfang Juli 1916 eröffnet. Vorläufer find, falls es überhaupt lolche gab, nicht feft= geftellt worden. Die Doft wurde zunächlt wohl in Oka= handja geftempelt. Mit dem Datum 1. September 1916 (Abb. 137) taucht der erfte Stempel in Tchwarzer Farbe 
auf. Klare Entwertungen find Tehr felten. Am 12. la= nuar 1917 war überhaupt nur noch das Datum des Stempels lesbar. Der Stempelkreis famt Ortsnamen und Stern war überhaupt nur noch eine Ichwarze un= leferliche Kleckferei. Zunächft verfuchte man fich zu behelfen. Mit dem Datum 23. Februar 1917 gibt es einen proviforifchen Stempel (Abb. 138), Es handelt fich zweifellos um den vorhergehenden Stempel, jedoch unter Hinzulatz neuer Typen. Die Farbe ift Tchwarz. Die Entwertung ift Tehr Telten!

Der endgiltige Stempel taucht zuerft im November 1917 auf, doch dürfte er Teinen Vorgänger Tchon weit eher abgelöft haben. (Ab. 139)

Die [chadhafte Geftalt des Stempels legt die Ver= mutung nahe, daß wir es mit einem alten deutrchen Stempel, etwa von Omaruru, zu tun haben, mit dem neu eingefeizten Namen "Waldaus. Diefer Stempel führt merkwürdigfte und verdrehte Daten, z. B. Itatt 13. 11. 17 die Zahl 19.7.13 und Ttatt 1.1.18 die Zahlen 19. 18. 8. Ferner kommt noch das Datum 19.7. $20 \mathrm{im}$ lahre 1918 vor. Die Farbe ift ein fchlechtes Graufchwarz.

\section{Walvis=Bay. (Walfilchbucht) poftagentur Brit. Belitzung.}

Die englifche Kültenbelitzung Walvis Bay, welche ganz ifoliert füdlich von Swakopmund im deutfchen Gebiete lag, unterftand vor dem Kriege der Verwaltung der füdafrikanilchen Union. Seit der Beletzung des Schutzgebietes wurde Walvis=Bay aus praktilchen Gründen der Regierung von Südweftafrika angegliedert.

Es waren nebeneinander 2 Stempel im Gebrauch (Abb. 140 und 147). Die Farbe ift [chwarz. Während der erfte Stempel durchwegs auf einfachen. Poftfachen vorkommt, wurde der zweite Stempel vorwiegend für Einfchreibebriefe verwandt. 


\section{Warmbad. poltamt}

Bezirk Warmbad.

Da der endgiltige Stempel erft früheftens im Herbft 1915 im Gebrauch kam, dürfte es Vorläufer geben. Die Farbe diefes Stempels (Abb. 142) ift ftets Ichwarz.

Vom 16. September, (warlcheinlich fchon 2-3 Wochen zeitiger) bis 29. Oktober 1916, war neben diefem Stempel der urfprüngliche deutlche mit veränderter Infchrift in Gebrauch. Auch leine Farbe ift Ichwarz, er ift recht Telten. (Ab. 143)

\section{Waterberg. \\ poltagentur. \\ Bezirk Omaruru.}

Der Eröffnungstag fällt gegen Ende 1915. Der einzige bekannte Stempel (Abb. 114) hat ftets Ichwarze Farbe.

\section{Wilhelmstal. \\ poltagentur \\ Bezirk Karibib.}

Es ift wahricheinlich, daß diefe Agentur bereits feit Anfang 1918 beftand, jedoch wurden die Briefe erft in Karibib abgeftempelt. Ein Stempel taucht zuerft mit dem Datum 29. Januar 1919 auf (Abb. 145). Es han= delt fich anfcheinend um den alten deut Chen Stempel, dellen Typen jedoch ftärkere Spuren der Abnützung aut= weifen. Die Farbe ift graulchwarz und meift fehr fleckig. 


\section{Windhuk. \\ Generalpoftamt. \\ Bezirk Windhuk.}

Am 12. Mai 1915 wurde Windhuk vom Feinde befetzt und wenige Tage fpäter das Doftamt eröffnet. Man bediente fich zuerft eines Kreisftempels ohne Orts= namen mit der Ziffer 6 im unteren Teil (Abb. 146). Die Farbe war grau und graufchwarz. Vom 19. Auguft, Ipäteftens 24. Auguft, findet fich ein intereflantes Fehl= datum vor. Statl der lahreszahl 15 nämlich 14. Der= artige Entwertungen find Tehr felten. Ende Auguft 1915 wurde diefer Stempel nach dreimonatlicher Kurs= dauer endgiltig eingezogen. Am 24. Juni war die Stempelfarbe violett (felten).

Gleichzeitig, aber nur vorübergchend, wurde der frühere deutfche Stempel (Abb. 147) mit unveränderter Infchrift benutzt. Bekannt ift eine einwandfreie Ent= wertung in Tchwarzer Farbe vorn 16. Juni 1915.

Für Einfchreibebriefe wurde zeitweilig in den erften Wochen ein großer Doppelovalltempel benutzt (Abb. 148) in violetter Farbe. Da diefer Stempel gleich= zeitig neben dem Stempel Abb. 146 auf Briefen vor= kommt, verdankt der Verfalter diefer Tatfache die Feft= ftellung eines Fehldatums. Während nämlich der einfache Stempel richtig auf den 29. Mai lautet, zeigt der Ovalftempel das Datum 26. Mai. Diefer Stempel (Abb. 148) kommt auf 2 Einfchreibebriefen vor, welche die Nummer 45 und 46 tragen. Weitere Abdrucke find dem Verfaller nicht bekannt geworden und dürfte der Stempel zu den größten Seltenheiten gehören.

Wăhrend diefe beiden Ziffernftempel noch in Ge= brauch waren, tauchte am 13. Augult 1915 ein endgil= tiger Stempel auf (Abb. 149). Es handelt fich um einen alten deutfchen Stempel, der noch die Schreibweife 
"Windhock" ftatt Windhuk aufweift. Der Landesname ift gänzlich entfernt. Die Farbe ift gewöhnlich lchwarz, wie bei allen folgenden Stempeln. Vom 13. Januar bis 23. lanuar 1916 finden wir ihn in dunkelvioletter Farbe auf eingefchriebenen Druckfachen. (Sehr felten.) Der Stempel kommt Tonft auch auf Einfchreibebriefen, Druck= Tachen wie auch als Ankunftsftempel vor.

Ende Auguft 1915 kam ein neuer englifcher Stem= pel in Gebrauch (Abb. 150). Auf gewöhnlichen Briefen findet man den Stempel nur bis Anfang März 1916, da= rauf wechlelweife auf Einfchreibebriefen und als $A_{n}=$ kunftsftempel.

Der endgiltige Stempel für gewöhnliche Briefe kommt ungefähr leit dem 9. März 1916 vor (Abb. 151). Die Farbe ift durchwegs [chwarz, jedoch wurde am 1. Juni 1916, vielleicht wenige Tage darauf noch, dunkel= violette Farbe beobachtet. UnregelmäBigkeiten, wie kopf= ftehende Daten, kommen häufiger vor.

NB. Keine eigentlichen Dofttempel find die Stem= pel Nr.152, 153 und 154 mit den Buchftaben R. L. S. (Re= giftered Letter Section) d. h. es lind die Windhuker $A n=$ kunfts- oder Durchgangsftempel für Einfchreibebriefe. Die Stempel haben entweder grüne, blaue, rote oder violette Farbe. Auf Marken können die Stempel nur zufällig vorkommen.

Auf Telegrammen findet man feit Ende luni oder luli 1915 Atändig den früheren deutfchen Stempel von Windhuk (Abb. 155) in Tchwarzer Farbe, jedoch ohne Landesnamen vor.

Vorübergehend war es erlaubt, nach Schalterfchluß um 5 Uhr nachmittags Briefe am Telegraphentchalter aufzugeben, die dann noch mit den Abendzügen be= fördert wurden. Solche Poftfachen wurden dann mit dem Stempel (Abb. 155) entwertet. Diefe Vergünftigung wurde aber bald wieder aufgehoben.

NB. Der deutfche Einfchreibezettel von Windhuk teils mit, teils ohne durchftrichenen alten Landesnamen kommt im März, Cowie Juni und luli 1916 vor. Derar= tige Briefe bedingen eine durchaus gerechtfertigte höhere Einfchätzung. 


\section{Witvley. (Gross=Witvley) \\ poltagentur. \\ Diftrikt Gobabis.}

Die Agentur wurde im Frühjahr 1916 eröffnet Vorläufer, wie handfchriffliche Entwertungen können vorkommen. Der erfte Stempel taucht mit dem Daium 25. September 1916 zuerft auf, dürfte jedoch anfdheinend bereits Ende Auguft benutzt Kein. (Abb. 156) Die Farbe ift fchwarz. Infolge rafcher Abnützung des Kaut fhuk= ftempels fielen die Entwertungen bereits nach halbjäh= rigem Gebrauch unleferlich aus. Deutliche Stempel find Tehr felten.

Der endgiltige Stempel wurde am 18. Mai 1918 zuerft gemeldet (Abb. 157). Die Farbe ift kräftig fchwarz. Da der urfprüngliche deutfche Ortsname diefer Station Groß Witvley lautete, to haben wir es natürlich mit zu= rechtgemachten Typen zu tun. Die Buchftaben $W$ und $y$ fcheinen von Anbeginn etwas befdaädigt zu Tein, 


\section{Bütow's}

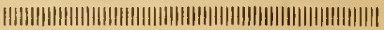

\section{großerMeßapparat}

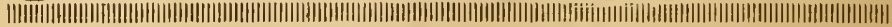

mit Nonius, Mikrometereinstellung und federnder Auflageplatte (siehe nebenstehende Abbildung)

ermöglicht

die Ablesung

von ganzen Milli=

metern und die genaue

Bestimmung der Bruchteile von ${ }^{1 / 20} \mathrm{~mm}$ ohne Schwierigkeiten.

Das Auge wird nicht - wie bei allen anderen Apparaten - angeftrengt,

denn durch die einfache Anwendung

des Nonius werden Bruchteile

des mm errechnet, nicht aber

abgelesen. Der Apparat

ist vollkommen

aus Messing

gearbeitet.

Preis 350.- Mark

Markenhaus und Philatelistischer Verlag GEORG BÜTOW, BERLIN C2 


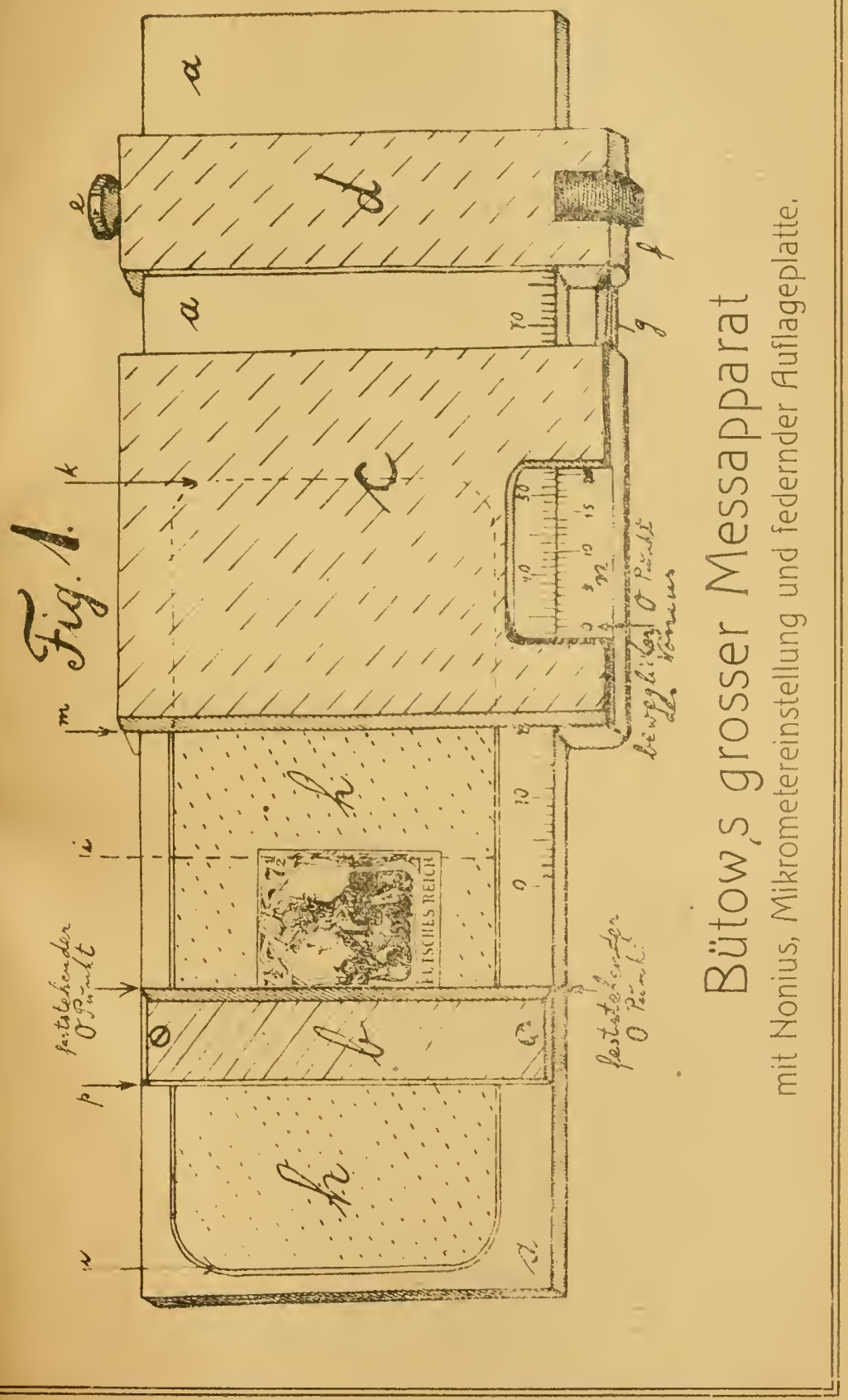




\section{Ppäzisions-Mlarsiala in Stall}

Modell 1920

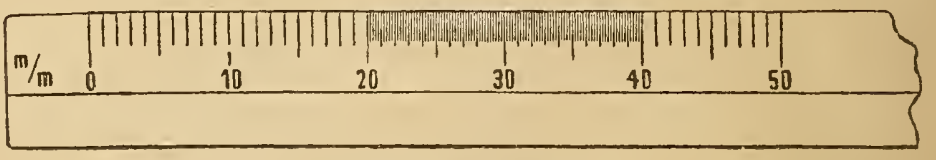

Stark vergrössert

Ohne Messingleiste Preis 12.-Mk. (Porto extra) Mit Griffleiste Preis 15.- Mark (Porto extra)

Der Meßstab soll für feine und feinste Längenmessungen dienen. Er besteht aus einer biegsamen schwedischen Stahlschiene, die in einem Messinghandgriff eingespannt ist. Die Teilung ist $5 \mathrm{~cm}$ lang. Hiervon sind die ersten 2 und der letzte $\mathrm{cm}$ in $\mathrm{mm}$, der dritte und vierte $\mathrm{cm}$ in fünftel $\mathrm{mm}$ greteilt. Die Teilstriche sind so fein eingeätzt, daß. sich die MeB-Ergebnisse auf einige Hundertstel mm abschätzen lassen. Die auf der Schiene befindlichen Teilstriche reichen bis unmittelbar an die Bildebene heran und lassen eine gen a u e Ablesung der gemessenen Längen zu.

Markenhaus und Philatelistischer Verlag Georg Bütow, Berlin C 2. 
Während die Teilstriche bei anderen Modellen eingeschlagen oder eingeritzt werden, sind dieselben bei vorstehendem neuen Modell eingeätzt, dadurch wird

1) eine grössere Feinheit der Teilstriche,

2.) eine grössere Genauigkeit der Maße erzielt.

Die Ausführung entspricht den höchsten Anforderungen an Genauigkeit und Sauberkeit.

In Gebrauch empfiehlt sich für alle diejenigen, die nicht ïber besonders scharfe Augen verfügen, die Anwendung unter gleichzeitiger Benutzung einer Lupe. Die Messung mit einem derartig feinen Anlege-Maßstab liefert bedeutend genauere Ergebnisse als die mit einem Glasauflege-Maßstab. Diese durchsichtigen Maßstäbe absorbieren viel Licht und haben außerdem meist ziemlich grobe Teilstriche, die in genaue Deckung mit der zu messenden Kante zu bringen sehr schwierig ist.

Das neue Meßins'rument, Modell 1920, eigneı sich besonders gut zum genauen Bestimmen der Länge von Marken, Aufdrucken, Abstempe= lungen. Es ist ein unentbehrliches Hilfsmittel für den Markenliebhaber und gehört auf den Arbeitstisch eines jeden Philatelisten.

Markenhaus und Philatelistischer Verlag Georg Bütow, Berlin C 2. 


\section{Deurscher}

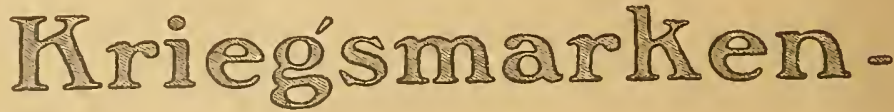
Sperial- Katล 1921

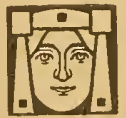

Neue Auflage erscheint im Frühjahr 1921

\section{Verlag:}

\section{Briefmarkenhandlung Geors Bütow} BERLIN, Burgstr. 27 (Bürohaus Börse) 


\section{Die deutschen}

\section{Kriegsmarken}

||||||||||||||||||||||||||||||||||||||||||||||||||||||||||||||||||||||||||||||||||||||||||||||||||||||||||||||||||||||||||||||||||||||||||||||||||||||||||||||||||||||||||||| von

Dipl.-Ing. W. Richter, Nürnberg

\section{1}

über 400 Seiten stark mit zahlreichen tabellarischen Aufstellungen, Bewertungen und einer Tafel Originalmarken

Das Buch erscheint in 7 Lieferungen, die erste als Doppel-Lieferung dürfte im April 1921 fertig gestellt sein, in weiterer etwa 6 wöchentlicher Folge erscheint der Rest

Preis der Doppel-Lieferung $35 \mathrm{Mk}$. jeder weiteren Lieferung $12 \mathrm{Mk}$. Porto besonders

PHILATELISTISCHER VERLAG, GEORG B UT OW BERLIN C 2, Burgstrasse 27 Postscheckk. 51810 


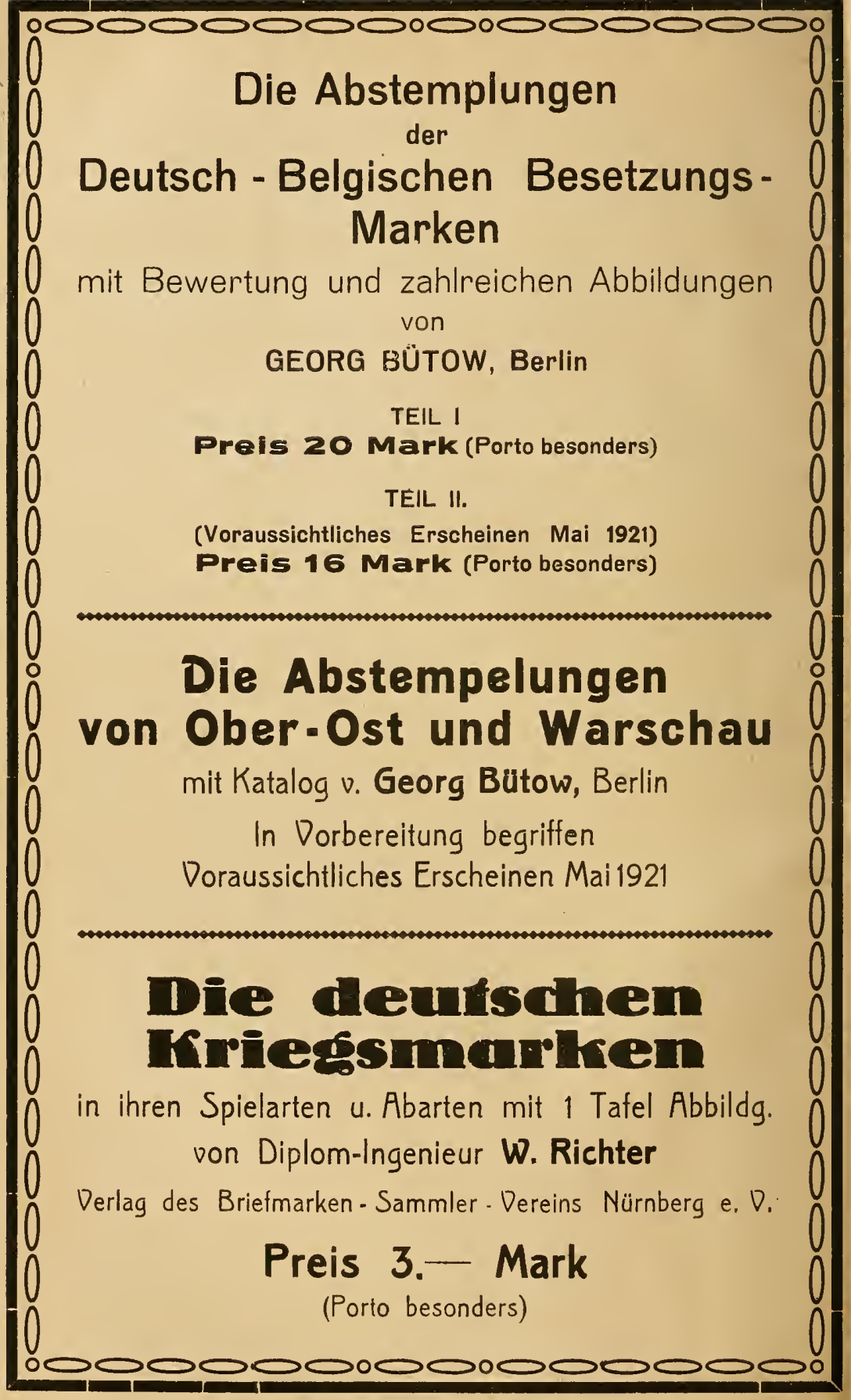




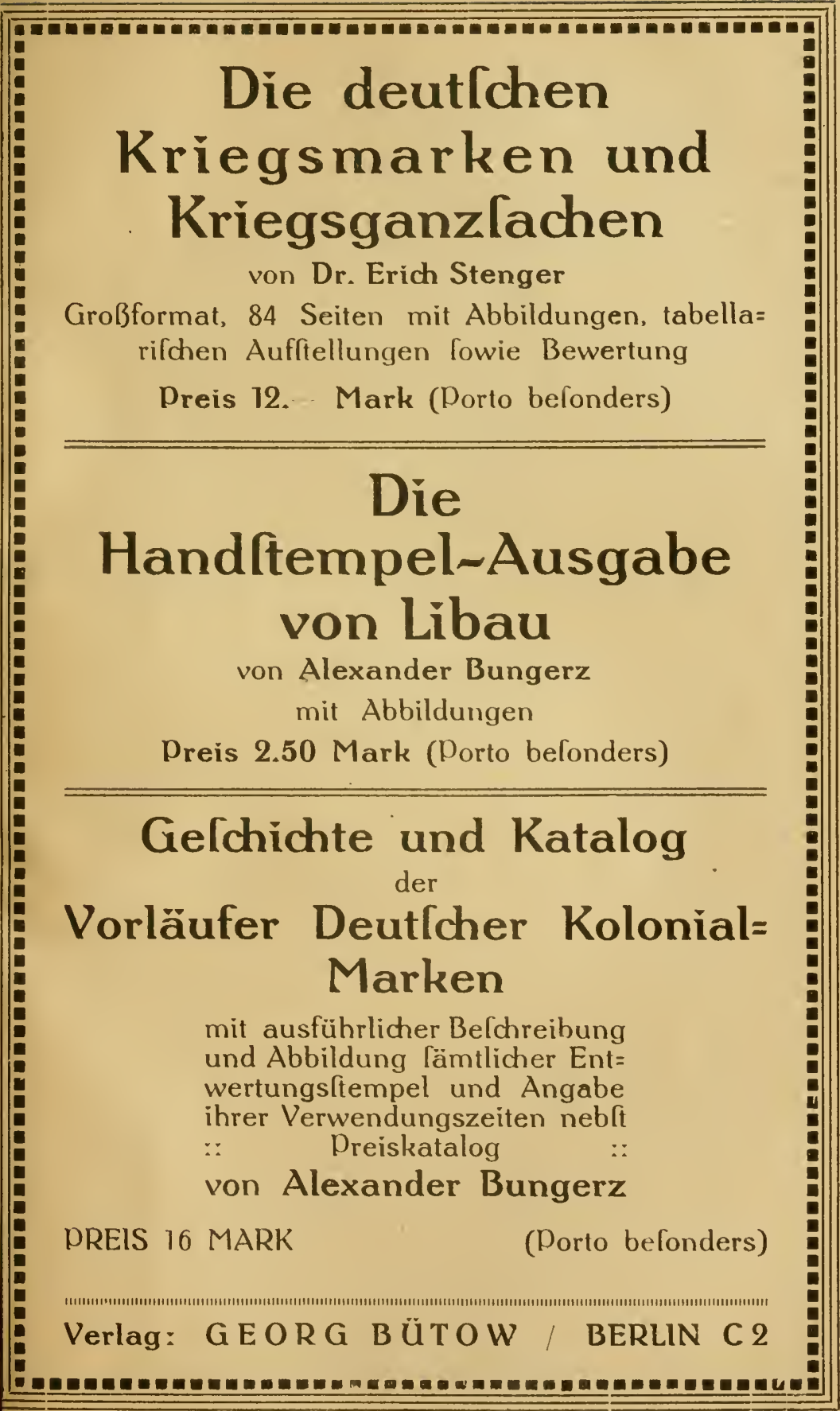




\section{Die Deutschen Feldpoststempel}

$1914 \ldots 1918$

von Hofrat Ferd. Meister, Nürnberg mit Bewertung

Preis 35 MK. Porto besonders.

\section{Die Manrirem \\ der Warselinar er Staditipest}

nebst einer Tafel Abbildungen der Marken, Aufdrucke und Entwertungsstempel von M. STEIN

Preis 3 Mk.

Porto besonders.

\section{Altbrasilien}

mit eirier Tafel Abbildungen v. Generalkonsul E. HEINZE

Preis 3 Mark

Porto besonders

Die Postwertzeichen der

\section{Republik Chile}

\section{von}

Dr.OTTO KRAUSE u. Dr. ARTHUR PHILIPPI

mit vielen Abbildungen und einer Karte von Chile Preis 30 Mark

Porto besonders 


\section{Die Postablagen-, Landpostboten-}

und Posthiltsstellen-Stempel von Bayern

Von Dr. ERICH STENGER und LUDWIG SAUTER Preis 15 Mark

Porto besonders

\section{0 jährige Geschichte der}

\section{bayerischen Postwertzeichen}

Von Dr. JOSEF HAYMANN und JOH. WILH. STUNDT, Nürnberg

Preis 6 Mark

Porto besonders

Die Bayerischen Feldpost-Stempel

mit vielen Stempelabdrïcken von Karl Holzschuher, Schwabach

= Preis 5 Mark $=$

Porto besorders

\section{AL T-BAYERN}

||||||||||||||||||||||||||||||||||||||||||||||||||||||||||||||||||||||||||||||||||||||||||||||||||||||||||||||||||||||||||||||||||||||||||||||

Abstempelungen

mit ausführlichem Katalog und

vielen Abbildungen

Von GEORG BUTOW, Berlin

In Vorbereitung begriffen. Voraussichtliches Erscheinen

Mitte 1921 


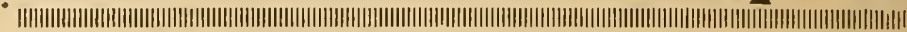

Historischer Katalog sämtlicher Luftposten

von

Dr. ROBERT PAGANINI, Zweisimmen (Schweiz) Preis 15 Mark

(Porto besonders).

\section{Geschichte \\ der Postwertzeichen von SERBIEN \\ $1866-1911$}

nach amtlichen Quellen von E. DEROCCO

PREIS Mk. 22.50

(Porto besonders)

In Vorbereitung:

Deutsches PhilatelistenAdreß-Buch 1921-1922

unter Mitwirkung der bedeutendsten Philatelisten-

Vereine zusammengestellt. 320 Seiten stark.

von

Rudolf Rohr, Berlin Preis 15 Mark (Porto besonders)

Voraussichtliches Erscheinen Mitte 1921 


\title{
Dänische Postfreimarken $1851-1901$
}

Historische Abhandlung von

O. KOEFOED, Kopenhagen

Mit amtlichen Neudruckbeilagen 150...- Mark Ohne Neudruckbeilagen 75.

Porto besonders

Die Postwertzeichen von

Norwegen

von R. STOEBE, Berlin

Preis 5 Mark

Porto besonders

\section{Die Postwertzeichen} des Grossfürstentums Finland

\author{
von L. HANCIAU \\ Preis 15 Mark \\ Porto besonders
}

\section{Typen und Abarten}

der Wendenschen Originalmarken,

Offiziellen Neudrucke

Nachdrucke und Fälschungen von W. Suenson

$$
\begin{gathered}
\text { Preis Mark } 22.50 \\
\text { Porto besonders }
\end{gathered}
$$




\section{Ein handliches PERMIIAIEEIII-ALLUIII}

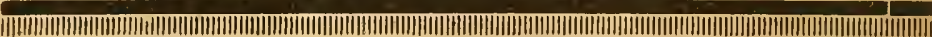

in Querformat $28 \frac{1}{2} \times 20 \mathrm{~cm}$ ohne Vordruck ist das von mir herausgegebene Spezial-Album. Dasselbe ist vornehm ausgestattet

es besteht aus losen Blättern, die $:::$ durch einen soliden $::$ : Selbstbinde-Rücken (mit Stahlklemmfedern) festgehalten werden. Durch eine n Handgriff kann der Selbstbinder abgenommen und sofort wieder angebrach werden. Ersatzbläller sind jederzeil nachzubeziehen. Das Album eignet sich zur Anlage von Spezialsammlungen vorzüglich, der $\mathrm{Be}-$ sitzer wird dauernd seine Freude haben.

Preis des Albums

mit 50 Blatt dickem Karton oder mit 50 Blatt dickem, re inw e i B e n Papier 60,- Mk. Einzelne Blätter je $50 \mathrm{Pf}$. Fassungsvermögen des Selbstbinders: 100 Blatt Papier oder 80 Blatt Karton. Farbe des Deckels: dunkelrot, dunkelblau, dunkelbraun oder dunkelgrün. Schutzfutteral (Karton) Stück. $1.50 \mathrm{Mk}$.

Markenhaus $u$. Philatelistischer Verlag Georg Butow, Berlin C2 Burg Strasse $27 \quad$ Fernspr.: Norden 2691 Bürohaus Börse 


\section{Suchen Sie}

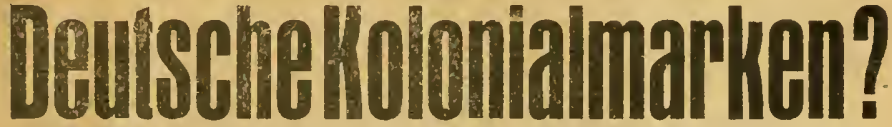

||||||||||||||||||||||||||||||||||||||||||||||||||||||||||||||||||||||||||||||||||||||||||||||||||||||||||||||||||||||||||||||||||||||||||||||||||||||||||| ungebraucht, gebraucht, auf Briefstiick,

Ganzbriefe, Abarten, seltene Abstempelungen, Streifen,

Blocks

Billigste Einzelwerte bis zu den $=$ grössten Seltenheiten $=$ dann verlangen Sie sofort die Auktionsbedingungen zu den

\section{viep |||||||||||||||||||||||||||||||||||||||||||||||||||||||||||||||||||||||||||||||||||||||||||||||||||||||||||||||||||||||

am 24. Februar, 24. Mai, 24. Septemb., 14. Dezemb.

der Firma

\section{Stuart Langford \& Co. BERLIN SW 48, Wilhelmstr. 113}


GEIR. WICHMAN N BERLIN NW6, KARLSTRASSE 13

Fachge[chäft für Meßinftrumente und andere

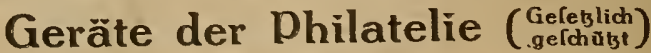

$1 / 2$ nat. Größe

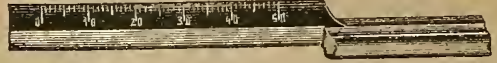
$1 / 2$ nat. Größe

Feinma 5 stab aus Metall, ftark verfilbert, Meßlänge $50 \mathrm{~mm}$, von $20-40$ in Fünftelmillimeter geteilt
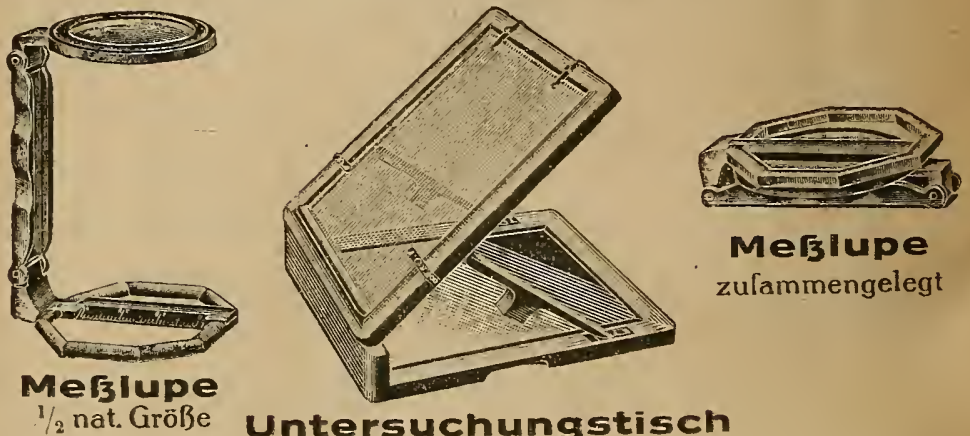

Me 3 lupe zulammengelegt

Untersuchungstisch

$1 / 10$ nat. Größe

Mefalupe mit Maßftab mit Fünftelmillimeterteilung, zufammen= klappbar.

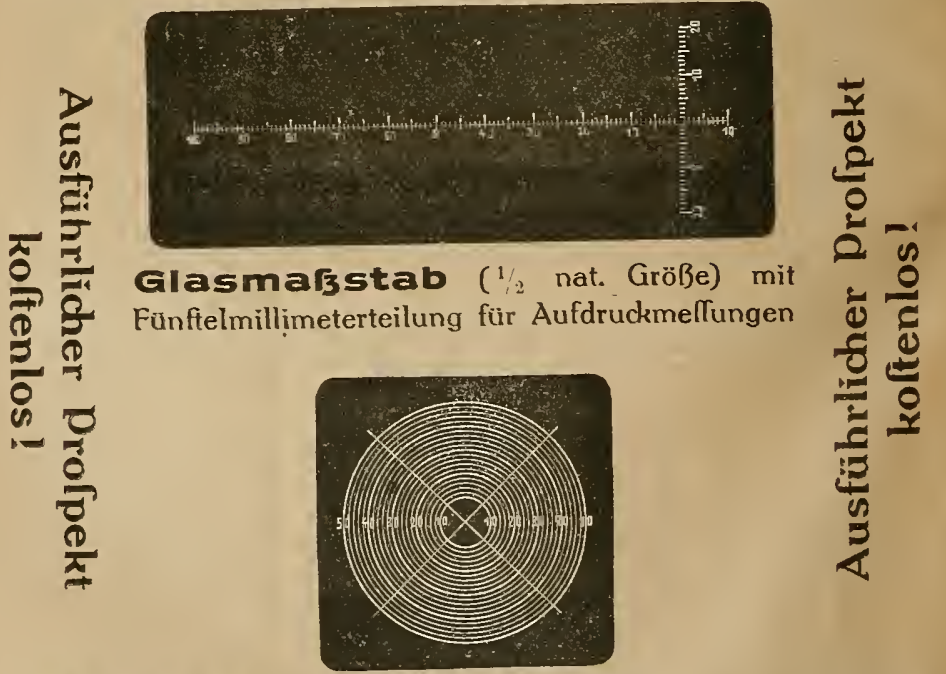

Kreismesser ( $1 / 2$ nat. Größe) aus Glas, für Stempelmeßungen. 


\section{Ein handliches}

\section{PERMAMEIIT-ALBUM}

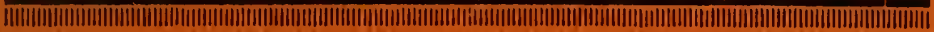

im Querformat $28 \frac{1}{2} \times 20 \mathrm{~cm}$ ohne Vordruck ist das von mir heraus. gegebene Spezial-Album. Dasselbe ist vornehm ausgestattet es besteht aus lo se n Blättern, die $:::$ durch einen soliden $\quad::::$ Selbstbinde-Rücken (mit Stahlklemmfedern) festgehalten werden. Durch e inen Handgriff kann der Selbstbinder abgenommen und sofort wieder angebracht werden. Ersalzhlaliter sind jederzell nachzubezlehen. Das Album eignet sich zur Anlage von Spezialsammlungen vorzüglich, der $\mathrm{Be}$ sitzer wird dauernd seine Freude haben.

Preis des Albums mit 50 Blatt dickem Karton oder mit 50 Blatt dickem, re i nw e i B e n Papier 60,-Mk. Einzelne Blätter je 50 Pf. Fassungsvermögen des Selbstbinders: 100 Blatt Papier oder 80 Blatt Karton. Farbe des Deckels: dunkelrot, dunkelblau, dunkelbraun oder dunkelgrün. Schutzfutteral (Karton) Stück $1.50 \mathrm{Mk}$.

Markenhaus u. Philatelistischer Verlag Georg Bütow, Berlin C2

Burg Strasse 27 Fernspr.: Norden 2691 Burohaus Borse 



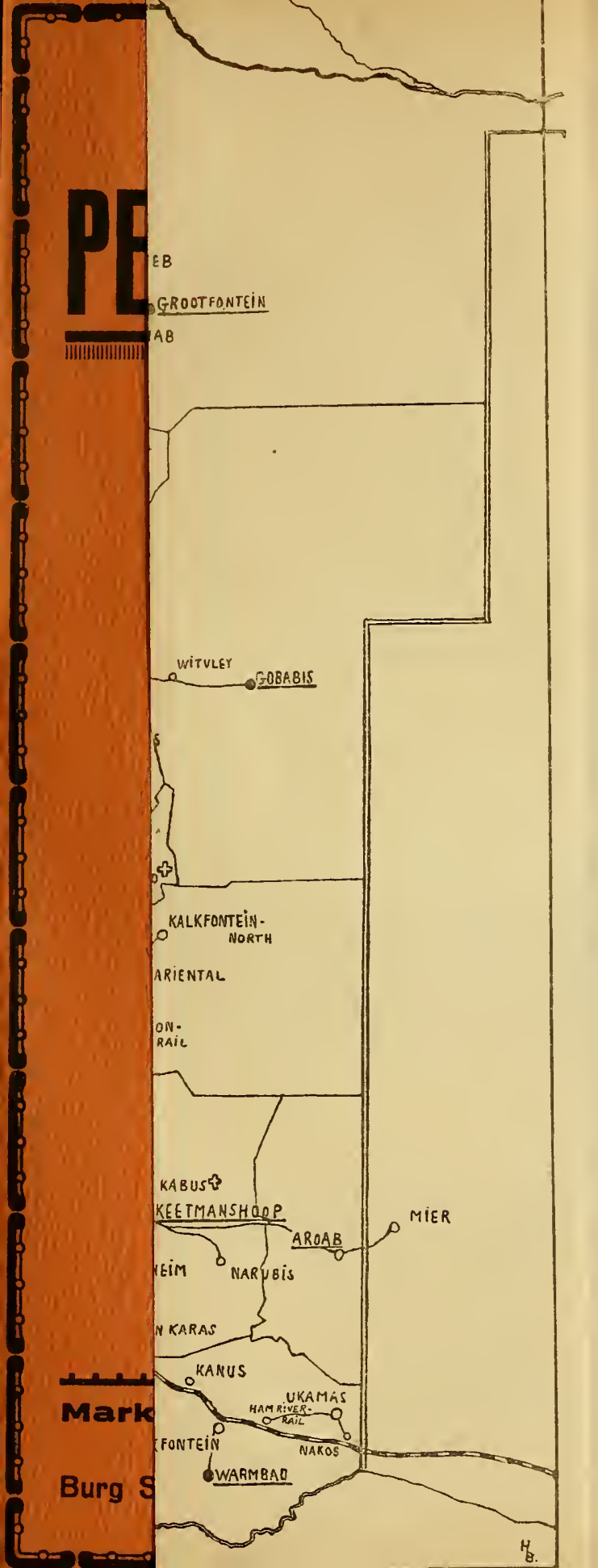





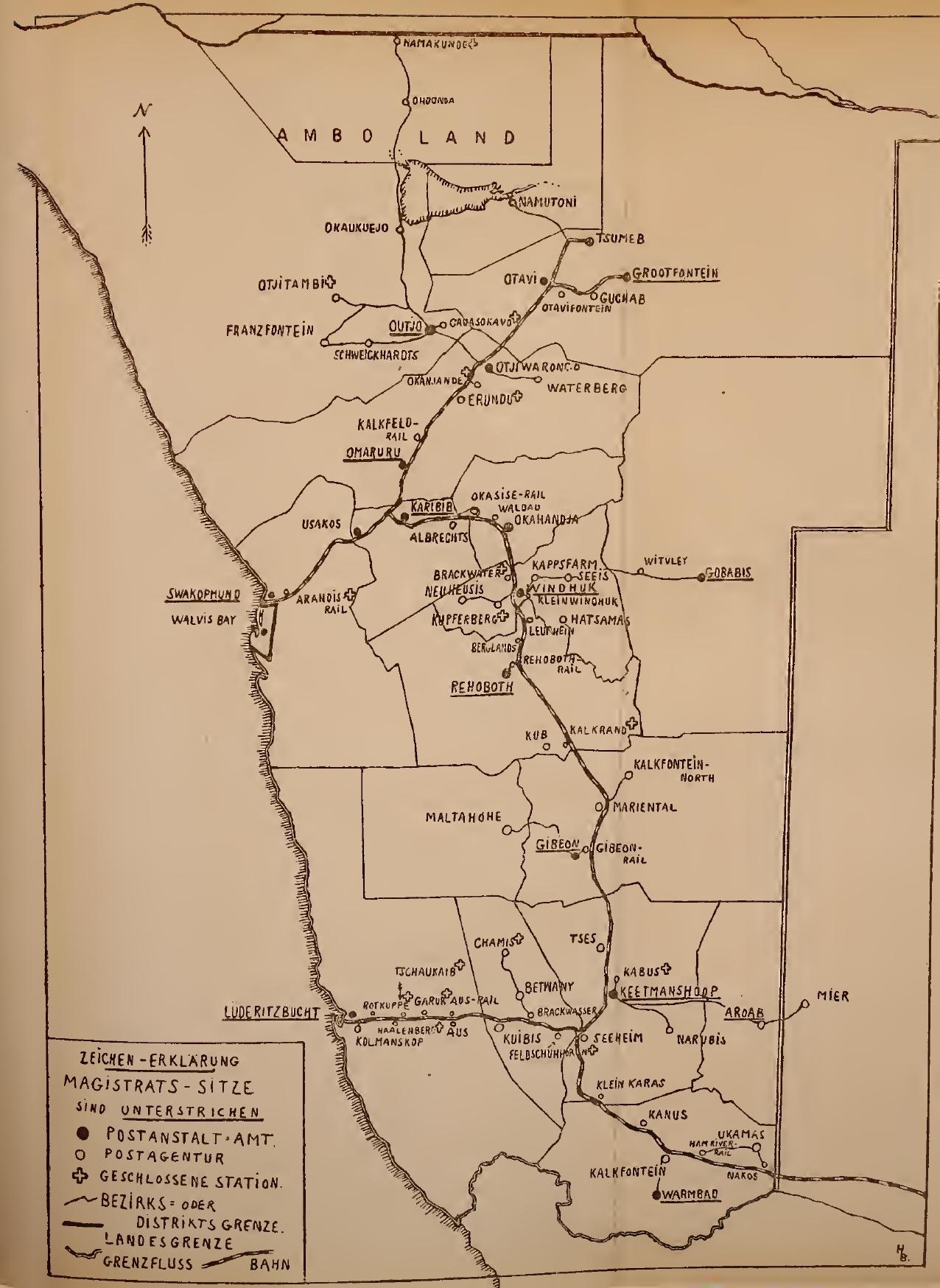




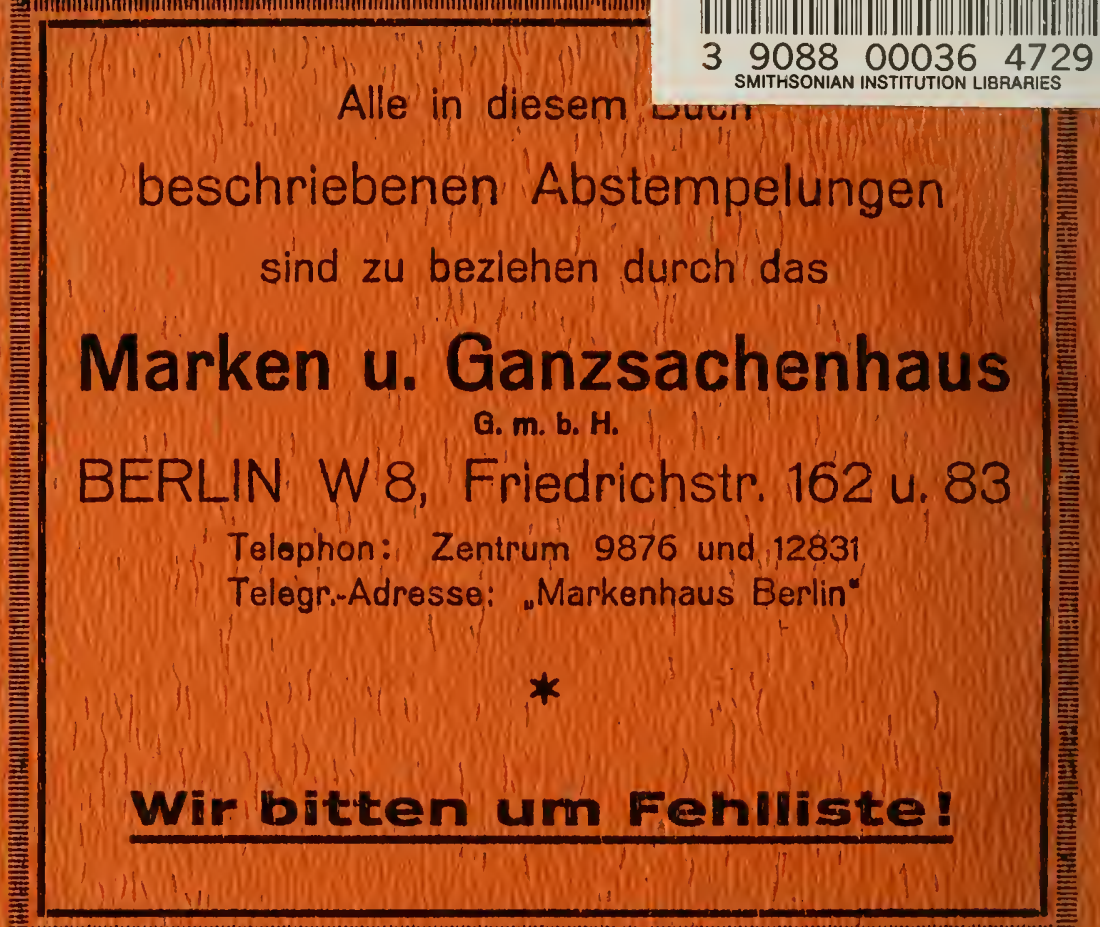

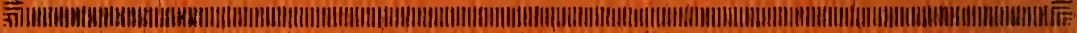

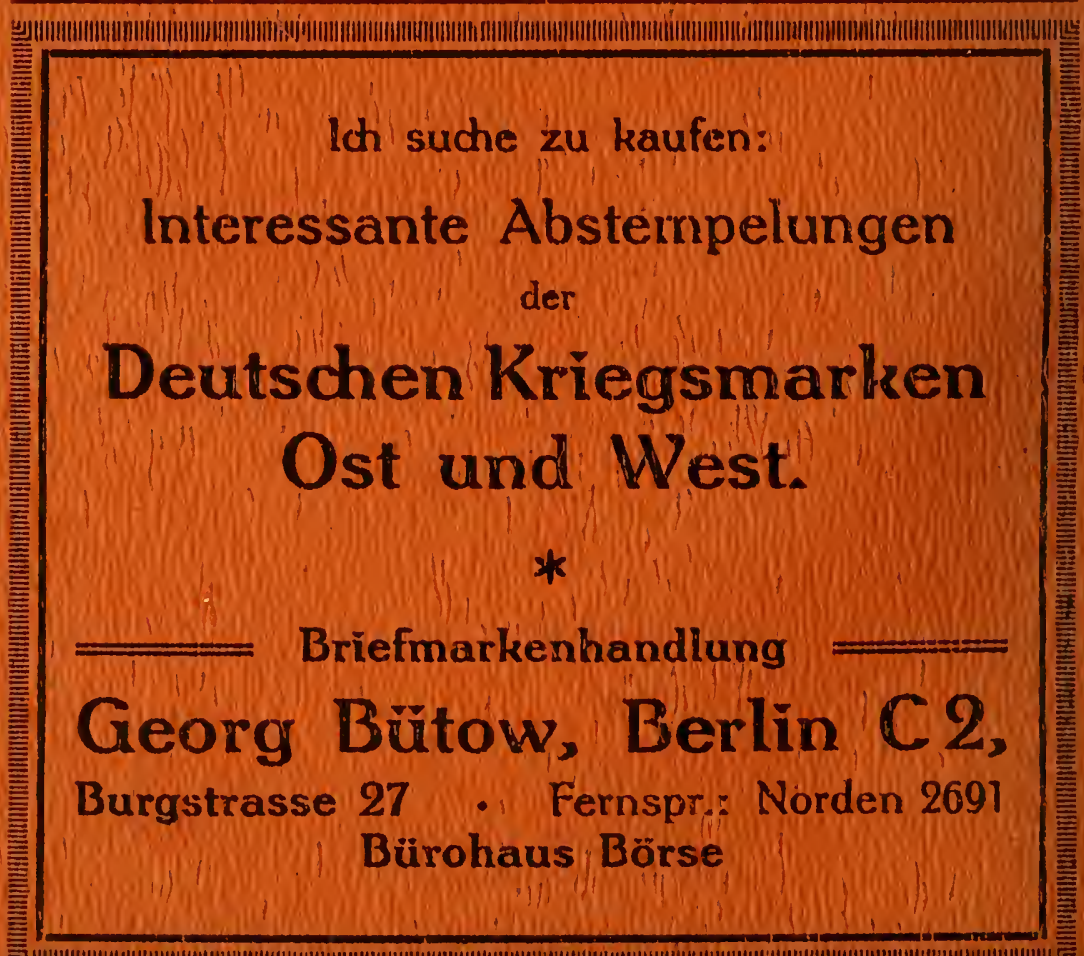

